\title{
Dynamics of alternative splicing during somatic cell reprogramming reveals functions for RNA-binding proteins CPSF3, hnRNP UL1, and TIA1
}

Claudia Vivori ${ }^{1,2,3}$ (D) Panagiotis Papasaikas ${ }^{1,4}$ (D), Ralph Stadhouders ${ }^{1,5}$ (D), Bruno Di Stefano ${ }^{1,6}$ (D) Anna Ribó Rubio ${ }^{1}$ (D),

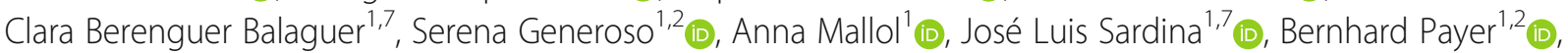
Thomas Graf ${ }^{1,2}$ (D) and Juan Valcárcel ${ }^{1,2,8^{*}}$ (D)

\footnotetext{
*Correspondence: juan.valcarcel@ crg.eu

'Centre for Genomic Regulation (CRG), The Barcelona Institute of Science and Technology, Carrer de Dr. Aiguader 88, 08003 Barcelona, Spain

${ }^{2}$ Universitat Pompeu Fabra (UPF), Carrer del Dr. Aiguader 88, 08003 Barcelona, Spain

Full list of author information is available at the end of the article
}

\begin{abstract}
Background: Somatic cell reprogramming is the process that allows differentiated cells to revert to a pluripotent state. In contrast to the extensively studied rewiring of epigenetic and transcriptional programs required for reprogramming, the dynamics of post-transcriptional changes and their associated regulatory mechanisms remain poorly understood. Here we study the dynamics of alternative splicing changes occurring during efficient reprogramming of mouse B cells into induced pluripotent stem (iPS) cells and compare them to those occurring during reprogramming of mouse embryonic fibroblasts.
\end{abstract}

Results: We observe a significant overlap between alternative splicing changes detected in the two reprogramming systems, which are generally uncoupled from changes in transcriptional levels. Correlation between gene expression of potential regulators and specific clusters of alternative splicing changes enables the identification and subsequent validation of CPSF3 and hnRNP UL1 as facilitators, and TIA1 as repressor of mouse embryonic fibroblasts reprogramming. We further find that these RNA-binding proteins control partially overlapping programs of splicing regulation, involving genes relevant for developmental and morphogenetic processes.

Conclusions: Our results reveal common programs of splicing regulation during reprogramming of different cell types and identify three novel regulators of this process and their targets.

Keywords: Alternative splicing, Somatic cell reprogramming, CPSF3, hnRNP UL1, TIA1, Pluripotency

(c) The Author(s). 2021 Open Access This article is licensed under a Creative Commons Attribution 4.0 International License, which permits use, sharing, adaptation, distribution and reproduction in any medium or format, as long as you give appropriate credit to the original author(s) and the source, provide a link to the Creative Commons licence, and indicate if changes were made. The images or other third party material in this article are included in the article's Creative Commons licence, unless indicated otherwise in a credit line to the material. If material is not included in the article's Creative Commons licence and your intended use is not permitted by statutory regulation or exceeds the permitted use, you will need to obtain permission directly from the copyright holder. To view a copy of this licence, visit http://creativecommons.org/licenses/by/4.0/. The Creative Commons Public Domain Dedication waiver (http://creativecommons.org/publicdomain/zero/1.0/) applies to the data made available in this article, unless otherwise stated in a credit line to the data. 


\section{Background}

Alternative splicing (AS) is a widespread mechanism of gene regulation that generates multiple mRNA isoforms from a single gene, dramatically diversifying the transcriptome (and proteome) of eukaryotic cells. Ninety-five percent of multi-exonic mammalian genes undergo AS, producing mRNA isoforms which often differ in coding capacity, stability, or translational efficiency and that can be translated into proteins with distinct structural and functional properties $[1,2]$. AS contributes to the regulation of many biological processes in multicellular eukaryotes, including embryonic development and tissue specification (reviewed in [3]). During the last decade, progress has been made to understand the role of post-transcriptional regulation (including AS) in the maintenance of cellular pluripotency and cell fate decisions, discovering genes differentially spliced between stem cells and differentiated cells and splicing regulators that control these choices [4-11].

During reprogramming into induced pluripotent stem (iPS) cells, somatic cells revert to a pluripotent state after overexpression of the transcription factors OCT4, SOX2, KLF4, and MYC (OSKM) [12]. Substantial progress has been made to understand the process at the transcriptional and epigenetic level, such as by identifying numerous roadblocks and some facilitators, but comparatively little is known about how post-transcriptional regulation impacts cell fate decisions. Recent work has revealed the functional relevance and conservation of splicing regulation during reprogramming [7, 13-16]. A conserved functional splicing program associated with pluripotency and repressed in differentiated cells by the RNA-binding proteins MBNL1 and MBNL2 was previously reported [7]. This splicing program includes a mutually exclusive exon event in the transcription factor FOXP1: a switch in inclusion of Foxp1 exons 16/16b modulates the functions of the transcription factor between pluripotent and differentiated cells [6]. Illustrating the complexity of such splicing program, dynamic changes of AS during cell reprogramming of mouse embryonic fibroblasts (MEFs) revealed sequential waves of exon inclusion and skipping in reprogramming intermediates and the functional role of splicing regulators in modulating reprogramming, in particular during the initial mesenchymal-to-epithelial transition (MET) phase [17]. Given the limited efficiency of cell reprogramming in this system, subpopulations of reprogramming intermediates had to be isolated through the expression of a pluripotency marker, biasing studies to the most prevalent and dominant factors.

Here we took advantage of the rapid, highly efficient and largely synchronous reprogramming of pre-B cells (hereafter referred to as "B cell reprogramming"), obtained by a pulse of the transcription factor C/EBP $\alpha$ followed by induced OSKM expression [18, 19] to study the dynamics of AS during this transition. The essentially homogeneous reprogramming of the cells in this system allowed detailed temporal transcriptome analyses of the bulk population, without the need of selecting for reprogramming intermediates. We established clusters of temporal regulation and compared these changes with the ones differentially spliced in MEF reprogramming [17]. Analyzing the dynamic expression of RNA-binding proteins (RBPs) during reprogramming, we inferred potential AS regulators, of which three were studied in detail. Characterization of these factors, namely CPSF3, hnRNP UL1, and TIA1, by perturbation experiments demonstrated their role as AS regulators in the induction of pluripotency. 


\section{Results}

Dynamics of alternative splicing in C/EBPa-enhanced B cell reprogramming occur independently from gene expression changes

To study the dynamics of changes in alternative splicing (AS) during cell reprogramming, primary mouse pre-B cells (hereafter referred to as "B cells") were reprogrammed as previously described [18-20]. Briefly, B cells were isolated from bone marrow of reprogrammable mice, containing a doxycycline-inducible OSKM cassette and an OCT4GFP reporter. These cells were infected with a retroviral construct containing an inducible version of $\mathrm{C} / \mathrm{EBP} \alpha$ fused to the estrogen receptor ligand-binding domain (ER). Infected cells were selected and re-plated on a feeder layer of inactivated MEFs. This was followed by a $18 \mathrm{~h}$-long pulse of $\beta$-estradiol, triggering the translocation of $\mathrm{C} / \mathrm{EBP} \alpha-\mathrm{ER}$ to the nucleus and poising the $\mathrm{B}$ cells for efficient and homogeneous reprogramming $[18,19]$. After washout of $\beta$-estradiol, reprogramming was induced by growing the cells for 8 days in reprogramming medium containing doxycycline (see "Methods" and [20]). RNA was isolated every other day from duplicates and subjected to paired-end sequencing (RNA-seq), resulting in high coverage (more than 100 million reads per sample) (Fig. 1A). As positive controls, mouse embryonic stem (ES) cells and induced pluripotent stem (iPS) cells were also sequenced.

AS analysis was performed using vast-tools [21], an event-based software that quantifies percent spliced in (PSI) values of annotated AS events and constitutive exons in all samples. These analyses revealed more than 14,000 AS changes during the entire reprogramming time course (for any possible pair of conditions: minimum absolute $\triangle$ PSI of 10 between PSI averages and minimum difference of 5 between any individual replicates across conditions) and a gradual increase in the number of differentially spliced events when comparing B cells to progressive stages of somatic cell reprogramming (Fig. 1B and Additional file 1: Figure S1A). Different classes of AS events were detected, with similar relative proportions at the various time points: $31-47 \%$ cassette exons, 5$11 \%$ alternative $3^{\prime}$ splice sites and $6-11 \%$ alternative $5^{\prime}$ splice sites, and $33-57 \%$ retained introns (Fig. 1B).

To classify the dynamics of AS changes during reprogramming, we selected cassette exon (CEx) events differentially spliced in at least one comparison (4556 exons) and performed a fuzzy c-means clustering analysis on their scaled PSI values (Mfuzz; [22]). This analysis revealed diverse kinetics of exon inclusion occurring during $\mathrm{B}$ cell reprogramming (Fig. $1 \mathrm{C}$, Additional file 1: Figure S1B-C and Additional file 2: Table S1). Six major clusters of AS dynamic profiles were detected: (1) exons that become included already after the C/EBP $\alpha$ pulse; (2) and (3) exons that are regulated either towards increased inclusion or skipping early after OSKM induction (day 2); (4) and (5) exons that display changes in inclusion at middle stages of reprogramming (day 4 onwards); (6) a cluster of exons only included at the latest steps of reprogramming (day 8 onwards) (Fig. 1C). Each of these clusters consisted of 300-500 exons. The inclusion levels of examples of exons belonging to different cluster types were validated by semi-quantitative RTPCR (Fig. 1D). For reference, changes in the PSI values of Grhl1 exon 6 and Dnmt3B exon 10, previously described to be associated with reprogramming and pluripotency $[17,23,24]$ were also quantified and found to follow similar inclusion 


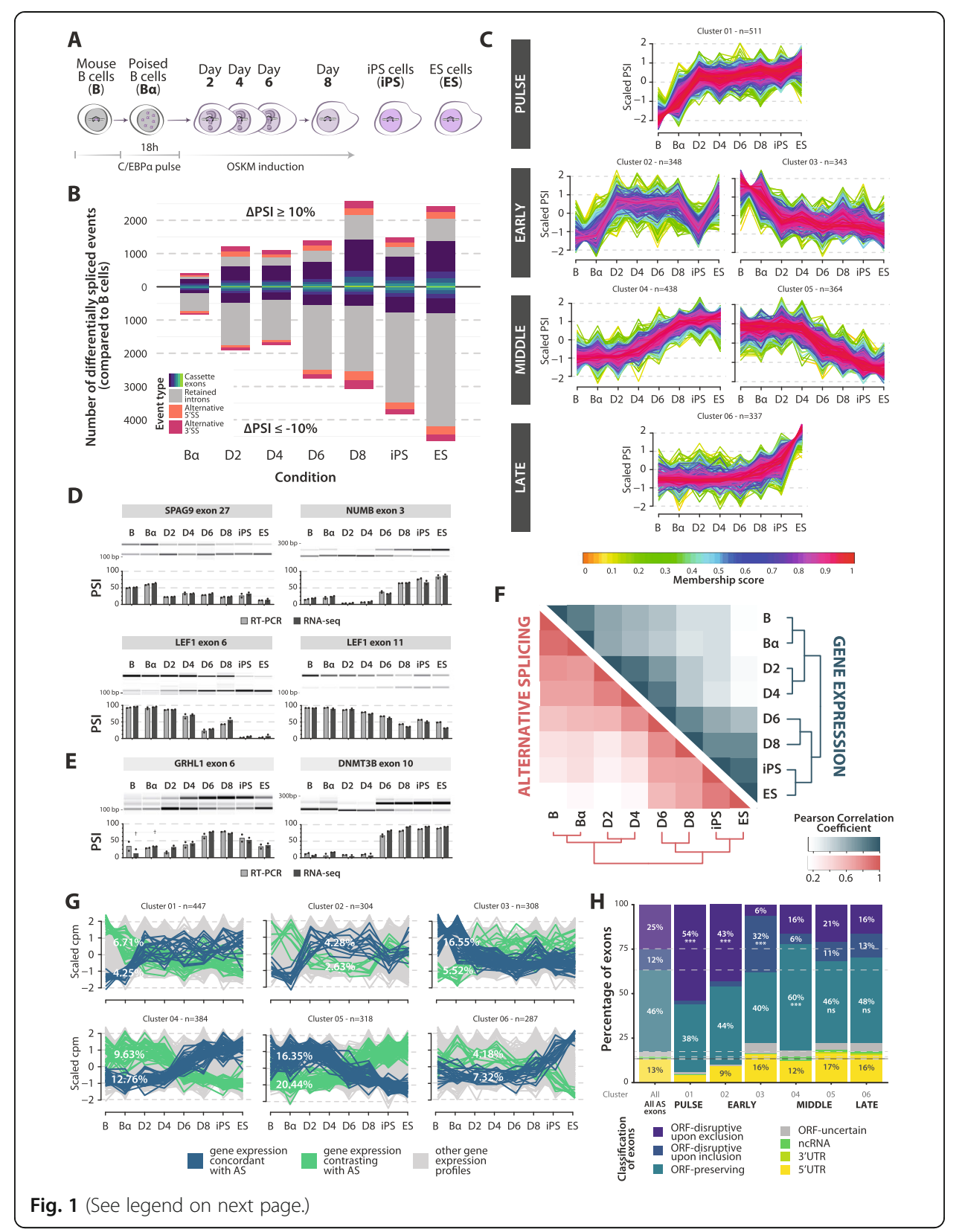


(See figure on previous page.)

Fig. 1 Dynamics of alternative splicing and gene expression changes during B cell reprogramming. A Schematic representation of C/EBPa-mediated B cell reprogramming time points and related controls analyzed by RNA-seq. Ba cells: B cells treated for $18 \mathrm{~h}$ with $\beta$-estradiol to activate C/EBPa. B Stacked bar plot representing cumulative number of events differentially spliced between B cells and subsequent reprogramming stages ( $x$-axis), as well as controls (iPS and ES cells). The $y$-axis represents the number of differentially spliced events compared to $B$ cells. The upper part corresponds to events with positive $\triangle \mathrm{PSI}$ values compared to B cells (> 10\%), the lower part to events with negative $\triangle P S I$ values compared to B cells $(<-10 \%)$. Red/orange areas: alternative 3'/5' splice sites (Alt3/Alt5) respectively; grey areas: retained introns; blue-green areas: cassette exons of increasing complexity (see "Methods"). See also Additional file 1: Figure S1A. C Clusters of cassette exons displaying related profiles of inclusion level changes during B cell reprogramming. Six clusters (out of a total of 12 identified, see Additional file 1: Figure S1B-C) are shown and classified into 4 categories, corresponding to the timing of the main shift observed (left). The $y$-axis represents scaled percent spliced in (PSI) values. The color of each line corresponds to the membership score of each exon relative to the general trend of the cluster. The size (number of events) of each cluster is indicated (n). D RT-PCR validation of selected cassette exon changes inferred from RNA-seq analyses. AS events assigned to different clusters were analyzed by semi-quantitative RT-PCR and quantified by capillary electrophoresis. Each panel includes a gel representation of the inclusion (top) and skipping (bottom) amplification products of one of the replicates and the corresponding quantification of the duplicates (PSI = molarity of inclusion product / molarity of inclusion + skipping products). Light grey columns: PSI values quantified by RT-PCR; dark grey columns: PSI values quantified by RNA-seq analysis using vast-tools software $(n=2)$. E Validation of Grhll exon 6 and Dnmt3b exon 10 inclusion level changes, previously associated with reprogramming and pluripotency, performed as in panel $\mathbf{D}$. Crosses indicate time points for which PSI values were calculated with low coverage (less than 10 actual reads). F Heatmap displaying correlations between B cell reprogramming stages according to gene expression (blue, top heatmap) and AS (red, bottom heatmap). Color scales represent Pearson correlation coefficient values calculated on the cpm values of the $25 \%$ most variably expressed genes or upon the PSI values of the $25 \%$ most variable cassette exons. G Gene expression patterns of genes containing the exons belonging to each of the AS clusters in panel $\mathbf{C}$. Genes with expression changes correlating with the cluster centroid or its negative (membership $>0.3$ ) are highlighted in blue and green, respectively, while the grey portion represents the (majority of) genes which follow gene expression profiles that do not match the changes in inclusion patterns of their exon(s). Percentage of concordant/contrasting patterns are displayed for each cluster. See also Additional file 1: Figure S1D. H Stacked bar plot representing the percentage of cassette exons in each of the AS clusters in panel $\mathbf{C}$ classified according to the following categories: disrupting the open reading frame (ORF) upon exclusion or inclusion, preserving the transcript ORF, mapping in non-coding RNAs, 3' UTRs or 5' UTRs or uncertain. The first column represents all exons differentially spliced between at least one pair of conditions, while the following ones represent the exons belonging to the each AS cluster (indicated below the bar). Fractions $<5 \%$ are not indicated. Where indicated, statistical significance was calculated by Fisher's exact test on the proportion of exons in the cluster compared to the general distribution of all AS exons $\left({ }^{*},{ }^{* *},{ }^{* * *}=p\right.$ value $<0.05,0.01,0.001$ respectively)

patterns in B cell reprogramming, compared to the ones previously described in other systems (Fig. 1E).

We next sought to compare general AS and gene expression dynamics during reprogramming. Gene expression was analyzed using the edgeR package [25] and the level of similarity between each pair of conditions was estimated using a Pearson correlation coefficient on the cpm (counts per million) values of the most variable genes (3rd quartile coefficient of variation, $n=2961$ ). In addition, Pearson correlation coefficient was calculated based on the PSI values from the most variable cassette exons (3rd quartile coefficient of variation, $n=1140$ ). Both analyses showed pronounced switches between days 4 and 6 post-OSKM induction (Fig. 1F). Overall, however, most clusters displayed matching profiles in gene expression and AS of any included exon in less than $10 \%$ of the genes, reaching a maximum of $20 \%$ in a subset of AS clusters (Fig. 1G and Additional file 1: Figures S1D-E). These results argue, as observed before in a variety of other systems (e.g., [26]), that global programs of regulation of gene expression and AS are uncoupled from each other.

Interestingly, a larger proportion of exons included (or skipped) at early stages of reprogramming are predicted to disrupt the open reading frame (ORF) of the transcripts upon exon skipping (or inclusion, respectively), compared to middle/late exons and to 
the general distribution of mapped alternative exons (Fig. 1H, classification as described in [21]). This suggests a higher impact of AS-mediated on/off regulation of the corresponding protein expression via nonsense-mediated decay (NMD), and a switch to expression of full-length proteins during early steps of reprogramming. Middle clusters, instead, contain more exons predicted to preserve the coding potential of their transcripts, implying modulation of the functions of their encoded protein isoforms rather than on/off switches (Fig. 1H). Consistent with these concepts, while PSI values of cassette exons tend to increase throughout reprogramming (Fig. 1B, blue bars), intron retention-generally leading to NMD—-tends to decrease in the course of reprogramming (Fig. 1B, grey bars).

\section{AS changes at intermediate reprogramming stages show commonalities with MEF reprogramming}

As a first step to identify key AS events and potential regulators important for reprogramming, we compared our transcriptome analysis of B cell reprogramming with that of MEF reprogramming [17]. The two datasets differ in the experimental design and time frame (compare Figs. 1A and 2A). Specifically, the MEF system required sorting of cells undergoing reprogramming using the SSEA1 surface marker, while this was not necessary for B cell reprogramming due to its efficiency. To facilitate the comparison between the two transcriptome datasets, vast-tools analysis was applied to the MEF dataset [17], which yielded 843 cassette exons differentially spliced $(|\Delta \mathrm{PSI}| \geq 10$, range $\geq 5$ ) between any pair of samples of the MEF reprogramming dataset. Despite differences in the experimental setup, $79 \%$ of these exons (669 out of 843) were also found to be differentially spliced in B cell reprogramming (Additional file 1: Figure S2A and Additional file 3: Table S2). A similar level of overlap was also observed at the level of other AS events (72\% of AS events in general, Additional file 1: Figure S2A). The overlap between differentially spliced exons in the two systems was higher for middle and late AS clusters than for early clusters of B cell reprogramming (Fig. 2B), as might be expected from the convergence on a common program of AS related to pluripotency.

To further compare the two datasets, we performed a Principal Component Analysis (PCA) on the gene expression of the most variable genes in both datasets (3rd quartile coefficient of variation, $n=2679$ ) separating the stages into four distinct groups by $k$ means clustering (Fig. 2C). These groups clearly distinguish between starting cells, early and late stages of reprogramming and pluripotent cells. The PCA allowed us to outline a "reprogramming pseudotime" which was subsequently used to select AS exons and regulators for functional characterization. It also further highlighted the transition between days 4 and 6 in B cell reprogramming, juxtaposing them with days 7/10 in MEF reprogramming. A heatmap displaying the scaled PSI values of the 669 common differentially spliced exons of the two datasets at the different steps of the reprogramming process shows substantial similarities in exon inclusion (Fig. 2D).

In contrast to the similarities in AS profiles between the two reprogramming systems, we observed that expression of RNA-binding proteins (RBPs) previously associated with pluripotency, somatic cell reprogramming, and/or development $[7,9,14,17]$ and presumably mechanistically linked to different aspects of post-transcriptional regulation, differed significantly between the two datasets (Fig. 2E). Despite these more divergent 
A Mouse Embryonic
Fibroblasts (Day 0)

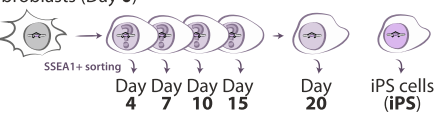

B

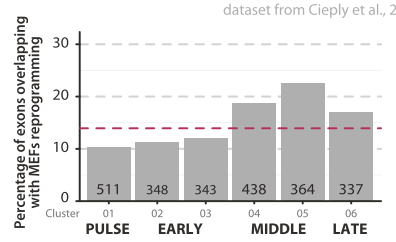

C

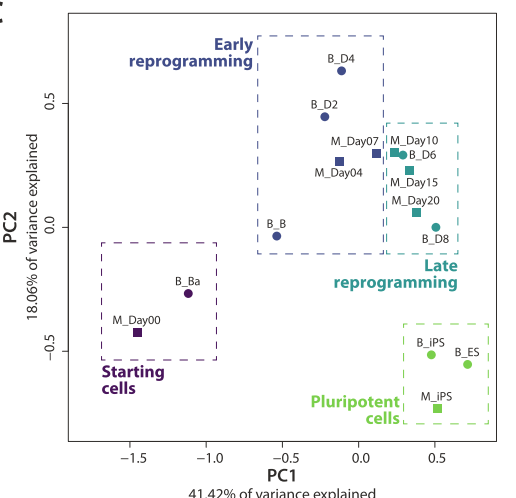

D

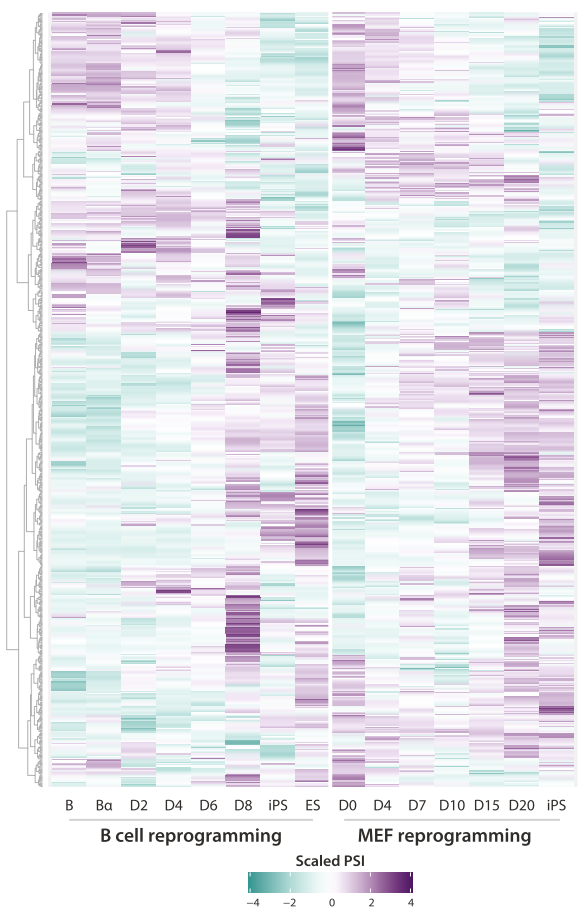

E

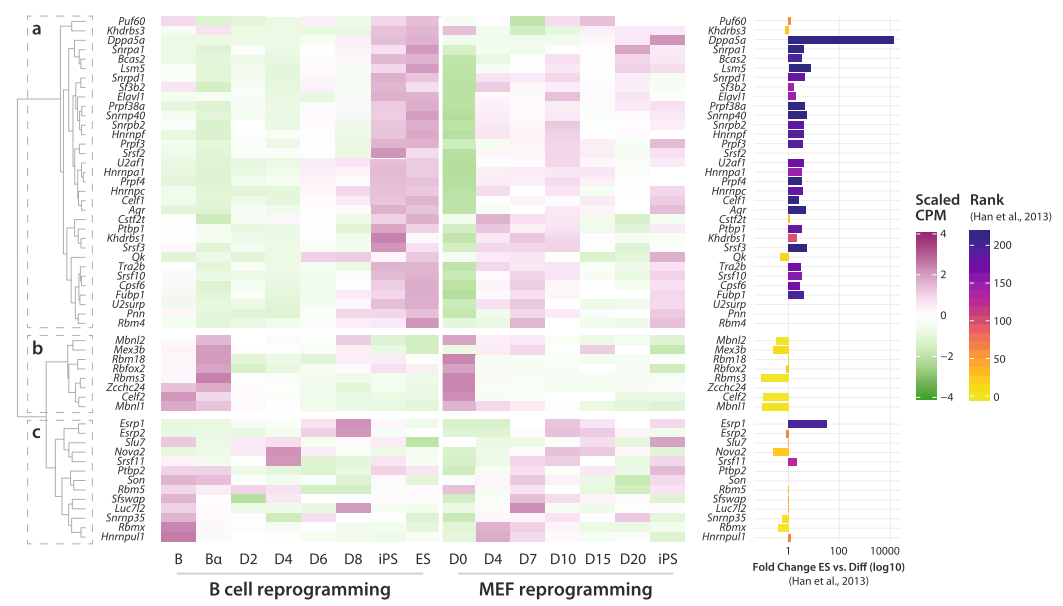

Fig. 2 B cell and MEF reprogramming systems share a program of AS changes. A Schematic representation of MEF reprogramming time points analyzed by RNA-seq in [17]. B Percentage of exons in each B cell reprogramming AS cluster that are also detected as differentially spliced in the MEF reprogramming dataset of [17]. The number of events in each cluster is indicated at the bottom of the corresponding bar. The magenta dashed line indicates the average percentage for all 12 clusters. C PCA analysis of the 25\% most variably expressed genes, segregated using $k$-means into 4 clusters: differentiated cells, early and late reprogramming and pluripotent cells, highlighted by colors and boxes. Circles: B cell reprogramming time points. Squares: MEF reprogramming time points. D Heatmap representing scaled PSI values (average between replicates) of exons differentially spliced in at least one time point in both B cell (left) and MEF reprogramming (right), with the corresponding hierarchical clustering. E Heatmap representing the expression of RNA-binding proteins known to be involved in pluripotency, somatic cell reprogramming, and/or development. Scaled cpm values (average between replicates) of both datasets are shown, with the corresponding hierarchical clustering. The bar plot (right) represents (when available) the fold change expression between ES cells and differentiated mouse tissues calculated in [7] and the corresponding ranking (color of the bar). 
profiles, hierarchical clustering captured three known functional groups, with cluster (a) containing factors with higher expression in iPS/ES samples (fold change $>0$ and high rank score according to 7, right panel) and known to promote pluripotency/reprogramming, such as $U 2 a f 1$ or $S r s f 2 / 3$ [9, 14]. In contrast, cluster $(b)$ contains factors with higher expression in the starting somatic cells, which includes known repressors of reprogramming such as Mbnl1/2, Celf2, and Zcchc24 [7, 11, 17]. Finally, cluster (c) contains factors with more variegated expression patterns at early and intermediate reprogramming steps (and mildly repressed in iPS/ES cells), including Esrp1/2 [16, 17] (Fig. 2E).

Taken together, our analyses revealed widespread AS changes during B cell reprogramming, which significantly overlapped with those of MEF reprogramming, especially at intermediate phases of the process.

\section{Predicted regulators of alternative splicing during somatic cell reprogramming}

To infer potential regulators of exons differentially spliced during B cell reprogramming, we extracted gene expression profiles of 507 RBPs (as annotated in the Uniprot database), which were detectably expressed (cpm $\geq 5$ in at least $33 \%$ of samples) and featured a minimum of variation across the $\mathrm{B}$ cell reprogramming dataset (coefficient of variation 20.2 ). Using the membership function of the $M f u z z$ package, we correlated (positively or negatively) the scaled gene expression profile of each RBP to each AS cluster centroid. This allowed us to derive a list of potential regulators whose changes in levels of expression correlate (or anti-correlate) with the profiles of AS changes in each cluster (membership >0.3) (Fig. 3, Additional file 1: Figure S3 and Additional file 4: Table S3). In line with previous work [7, 11, 14], our analysis identified known AS regulators involved in the induction or repression of pluripotency/developmental decisions, such as Mbnl1/2, Celf2 (both potential negative regulators of pulse/late clusters $1 / 6$ ), and U2af1 (potential positive regulator of middle cluster 4) (Fig. 3). Importantly, additional RBPs and splicing factors without previously known functions in reprogramming emerged as possible regulators.

To further define such regulators, we focused on RBPs that change their expression after the C/EBP $\alpha$ pulse or at early stages of reprogramming for functional validation during the induction of pluripotency, as we speculated that these could drive the inclusion/skipping of both early and intermediate AS exons during the reprogramming of $\mathrm{C} / \mathrm{EBP} \alpha$-poised B cells. We selected CPSF3 as a putative positive regulator of very early events because its profile of expression increases during $B$ cell and MEF reprogramming in parallel with centroid of AS changes in cluster 1 (Fig. 3A). While CPSF3 was originally described as part of cleavage / polyadenylation complexes [27], more recent work implicated components of this complex on alternative splicing regulation, including numerous internal exons not linked to the selection of alternative polyadenylation sites [28, 29]. Following a similar logic, we chose two putative negative regulators of cluster 1, namely TIA1, a well-described AS regulator with roles in cell proliferation and development (see below), and hnRNP UL1, a member of the heterogeneous ribonucleoprotein (hnRNP) family whose involvement in splicing regulation is largely unexplored (Fig. 3B). Due to experimental difficulties of genetic manipulation of the B cell reprogramming system (see "Discussion"), we modulated their expression at early 

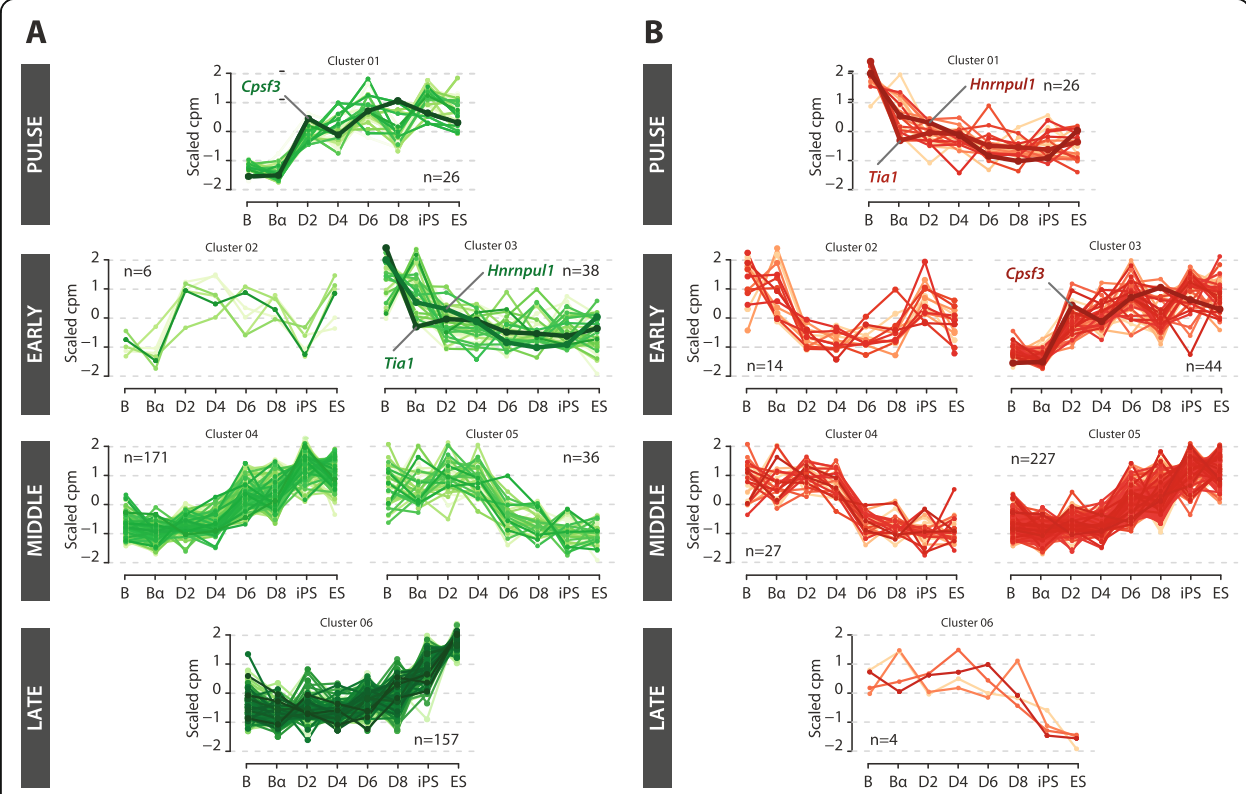

Fig. 3 Inferred regulators of AS changes during B cell reprogramming. A Gene expression profiles of RNAbinding proteins (RBPs) correlating with the centroid of each AS cluster (positive regulators). Average scaled cpm values are represented by each line and the number of putative positive or negative regulators of each cluster is indicated (1). Expression profiles of Cspf3, Hnrnpul1 and Tial are highlighted. See also Additional file 1: Figure S3A. B Gene expression profiles of RBPs correlating with the negative of the centroid of each AS cluster (negative regulators). Displays as in panel A. See also Additional file 1: Figure S3B

stages of MEF reprogramming [30] and examined the consequences on the dynamics of pluripotency induction and on relevant AS alterations.

\section{Knockdowns of CPSF3 or hnRNP UL1 repress MEF reprogramming}

The Cleavage and Polyadenylation Specificity Factor (CPSF) complex is primarily involved in mRNA polyadenylation, but a number of its components, including CPSF3, has been shown to also play a role in splicing [28, 29, 31-33]. Cpsf3 expression increased early during reprogramming of both $\mathrm{B}$ cells and MEFs (Figs. 3A and 4A). The expression of Hnrnpul1, an hnRNP whose function in RNA metabolism is poorly understood, decreases in $\mathrm{B}$ cells after the $\mathrm{C} / \mathrm{EBP} \alpha$ pulse and then stabilizes at levels similar to those observed throughout MEF reprogramming (Figs. 3B and 4B).

To test their effects on MEF reprogramming, two different short hairpin RNAs (shRNAs) for each factor were cloned into lentiviral vectors containing a GFP reporter. The protocol used is summarized in Additional file 1: Figure S4A. Briefly, early passage MEFs isolated from reprogrammable mice were transduced with constructs bearing the shRNAs and the cells treated with doxycycline to activate OSKM (day 0). GFP ${ }^{+}$cells were FACS-sorted $48 \mathrm{~h}$ post-infection and seeded on inactivated MEFs serving as feeder layers, to proceed with reprogramming for up to 14 days. Cells were harvested every other day and samples analyzed by RT-qPCR for the expression of the shRNAs target and of pluripotency markers, and by flow cytometry to quantify the proportion of cells expressing the early pluripotency cell surface marker SSEA1 (appearing around days 56) and the later pluripotency marker EPCAM1 (appearing around day 8, [34]). At day 


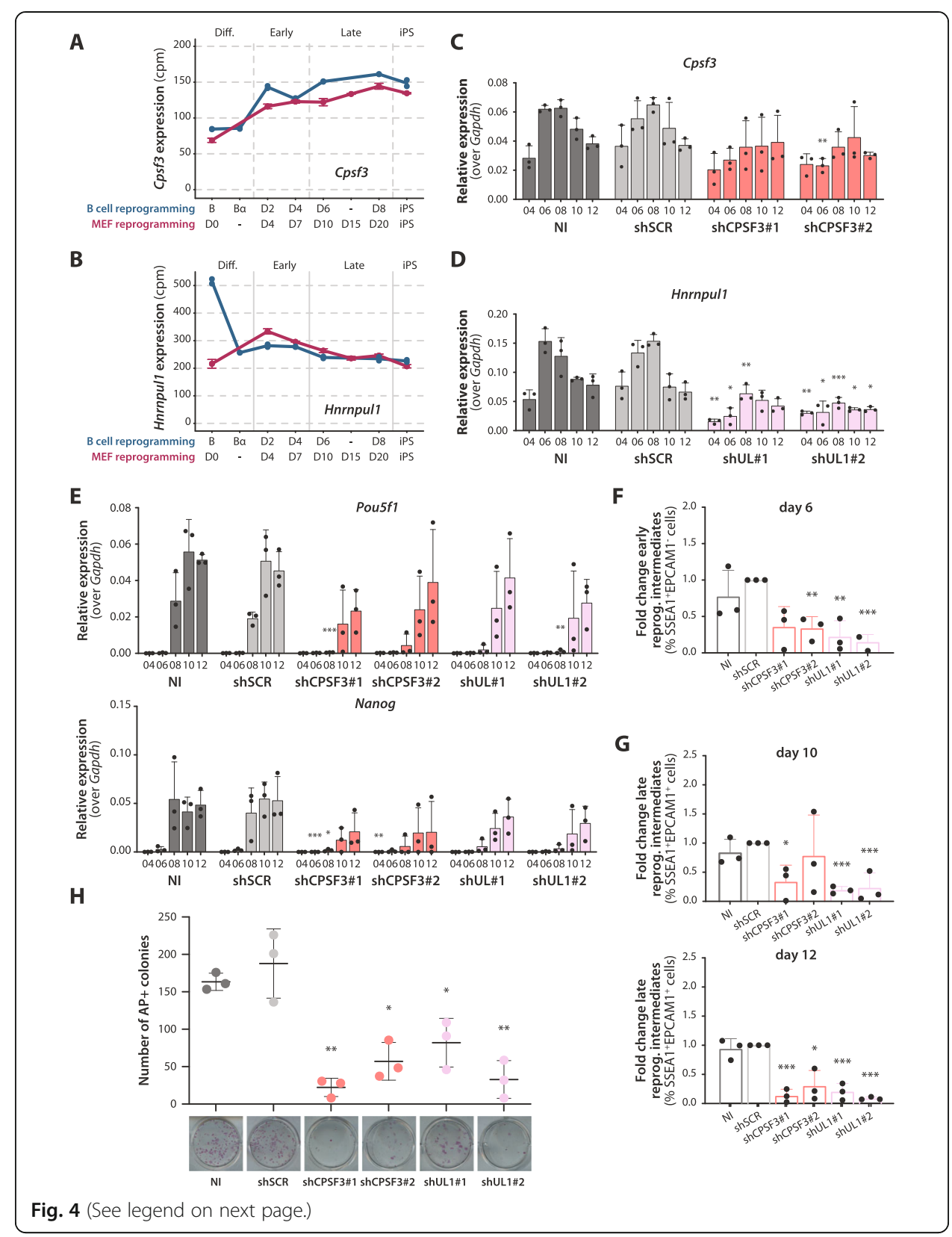


(See figure on previous page.)

Fig. 4 Knockdowns of CPSF3 or hnRNP UL1 impair MEF reprogramming. A Gene expression profiles of Cpsf3 in B cell reprogramming and MEF reprogramming (cpm values, blue and magenta lines, respectively). The $x$-axis represents "reprogramming pseudotime" in both datasets, calculated through the PCA analysis of Figure 2B. B Gene expression levels of Hnrnpul1 in B cell and MEF reprogramming (cpm values, blue and magenta lines respectively), as in panel $\mathbf{A}$. C Relative expression levels of Cpsf3 mRNA quantified by RTqPCR in non-infected cells (NI), cells transduced with a scrambled control shRNA (shSCR) or one of two shRNAs specific for Cpsf3. The $y$-axis represents the relative expression $(2 \wedge(-\Delta C t)$ value) of Cpsf3 after normalization over Gapdh. D Relative expression levels of Hnrnpul1 mRNA quantified by RT-qPCR as in panel C. E Relative expression levels of Pou5f1 and Nanog quantified by RT-qPCR as in panels C and D. See also Additional file 1: Figure S4B. B-E Average of biological triplicates and SD values are shown. Statistical significance was calculated by $t$-test on $\Delta \mathrm{Ct}$ values compared to the $\mathrm{NI}$ control $\left(*^{*},{ }^{*},{ }^{* * *}=p\right.$ value $<0.05$, 0.01, 0.001 respectively, corrected for multiple testing using Holm-Sidak method). F Reduction of early reprogramming intermediates at day 6 post-OSKM induction upon knockdowns of Cpsf3 and Hnrnpul1. Fold change was calculated from the percentage of SSEA1+EPCAM1- cells (of the total of alive cells) in every condition compared to the shSCR control using flow cytometry analysis. G Reduction of late reprogramming intermediates at days 10 and 12 post-OSKM induction upon knockdown of Cpsf3 or Hnrnpul1. Fold change was calculated from the percentage of SSEA1+EPCAM1+ cells (of the total of alive cells) in every condition compared to the shSCR control using flow cytometry analysis. See Additional file 1: Figure S4C for examples of gates. $\mathbf{H}$ Number of colonies stained with alkaline phosphatase (AP) at day 14 post-OSKM induction upon knockdown of Cpsf3 or Hnrnpul1. On the bottom, images of representative wells are shown for every condition. F,G,H Average of biological triplicates and SD values are shown. Statistical significance was calculated by $t$-test comparing each condition to the shSCR control $\left(^{*},{ }^{* *},{ }^{* * *}=p\right.$ value $<0.05$, $0.01,0.001$ respectively, corrected for multiple testing with Holm-Sidak method)

14, 2 days after removing doxycycline, cultures were stained for alkaline phosphatase (AP) activity to identify iPS colonies and to assess the efficiency of reprogramming.

Cells infected with shRNAs targeting Cpsf3 and Hnrnpul1 were compared with noninfected (NI) cells, as well as with cells transduced with a scrambled control shRNA (shSCR). Knockdown efficiency, quantified by RT-qPCR (Fig. 4C, D), showed a $51 \%$ and $58 \%$ reduction in Cpsf3 at day 6 post-infection with shCPSF3\#1 and \#2, respectively, becoming slightly less efficient during reprogramming (Fig. 4C). Similarly, knockdown of Hnrnpul1 resulted in $81 \%$ and $76 \%$ reduction of mRNA levels at day 6 postinfection with shUL1\#1 and \#2, respectively (Fig. 4D). The increase in the mRNA levels of endogenous Pou5f1 (encoding OCT4) and Nanog pluripotency markers was delayed in both Cpsf3 and Hnrnpul1 knockdown cells (compare for example values at day 8 in Fig. 4E and Additional file 1: Figure S4B), suggesting slower reprogramming kinetics. Consistent with these observations, knockdown of Cpsf3 and Hnrnpul1 reduced the percentage of cells expressing SSEA1 at day 6 (Fig. 4F) and cells expressing both SSEA1 and EPCAM1 at days 10-12 (Fig. 4G and Additional file 1: Figure S4C). Survival of cells during reprogramming did not seem to be affected in the knockdowns because no significant increase in the proportion of DAPI-stained cells was observed throughout reprogramming (Additional file 1: Figure S4D). Finally, we counted the number of AP positive colonies at day 14 and found that the amount was significantly reduced in cells infected with either the shRNAs targeting Cpsf3 or those targeting Hnrnpul1 (Fig. 4H). We finally tested the effects of overexpression of a T7-tagged version of CPSF3 from the beginning of reprogramming (Additional file 1: Figure S4A and E). A trend towards increased expression of Pou5f1 and Nanog at late times of reprogramming upon T7Cpsf3 overexpression was observed (Additional file 1: Figure S4F). However, this was not accompanied by enhanced reprogramming efficiency or redistribution of reprogramming intermediates (Additional file 1: Figure S4G-I), likely because of additional 
rate-limiting steps required to increase the efficiency of this complex process or because of suboptimal timing or levels of overexpression achieved in the experiment.

Taken together, these data show that the knockdown of Cpsf3 or Hnrnpul1 reduces the MEF to iPS reprogramming efficiency, therefore arguing that both RBPs contribute to the induction of pluripotency.

\section{Overexpression of TIA1 represses MEF reprogramming}

TIA1 is an RBP and AS regulator [35-37]. Tia1 mRNA levels decrease early during B cell reprogramming (Figs. 3B and 5A), compatible with a potential role as a repressor of cell reprogramming in this system. Because depletion of TIA1 induces mouse embryonic lethality and the protein is important for MEF proliferation, cell cycle progression, autophagy, and numerous signaling pathways [38], we decided to test the effects of TIA1 overexpression during MEF reprogramming. For this purpose, primary MEFs were infected at day 0 (concomitantly with the induction of reprogramming), with retroviral constructs containing a T7-tagged Tia1 cDNA and a GFP reporter to allow sorting of the transduced cells (Additional file 1: Figure S4A). Levels of Tia1 were quantified by RT-qPCR (24-fold increase compared to cells transduced with an empty vector, Fig. 5B). Consistent with our prediction from its expression profile in B cell reprogramming, overexpression of Tia1 repressed the induction of endogenous Pou5f1 and Nanog genes (Fig. 5C and Additional file 1: Figure S5A) and led to a reduction of early SSEA $1^{+} \mathrm{EPCAM}^{-}$cells and of late reprogramming intermediates (SSEA1 ${ }^{+} \mathrm{EPCA}$ $\mathrm{M}^{+}$cells) compared to empty vector and non-infected controls (Fig. 5D, E and Additional file 1: Figure S5B). In addition, it significantly reduced the count of $\mathrm{AP}^{+}$colonies at day 14 post-OSKM induction compared to controls (Fig. 5F), without substantially affecting the viability of reprogramming cells (Additional file 1: Figure S5C). Of note, overexpression of Tia1 delayed the gradual skipping of Lef1 exon 6, a conserved AS event between B cell and MEF reprogramming (Fig. 5G), which might partially explain the observed reduction in reprogramming efficiency.

Taken together, the observed reduced expression of TIA1 during B cell reprogramming and its impairment of MEF reprogramming when overexpressed suggest that it functions as a general repressor of pluripotency induction.

\section{CPSF3, hnRNP UL1, and TIA1 regulate alternative splicing during reprogramming}

To assess the effects of CPSF3, hnRNP UL1, and TIA1 manipulation on AS during reprogramming, RNA-seq analyses were carried out with RNAs isolated from MEFs at 0 and at 12 days post-OSKM induction, comparing the effects of Cpsf3/Hnrnpul1 knockdown (two different shRNAs for each factor) or Tia1 overexpression with those of the corresponding shSCR/empty vector controls (Fig. 6A). Quantification of gene expression of Tia1, Cpsf3, and Hnrnpul1 at the two timepoints is shown in Additional file 1: Figures S6A-B.

To determine the impact of these perturbations on AS, we calculated differentially spliced events between day 0 and day 12 in each condition as described before ( $\mid \Delta \mathrm{PSI}(-$ day12 - day0) $\mid \geq 10$, range $\geq 5$ ). TIA1-dependent events were defined as those changing during reprogramming only in the control or the overexpression condition, with $\mid \Delta \Delta$ PSI(TIA1 - Empty) $\mid \geq 10$ (colored dots in Fig. 6B). Similarly, CPSF3-dependent 


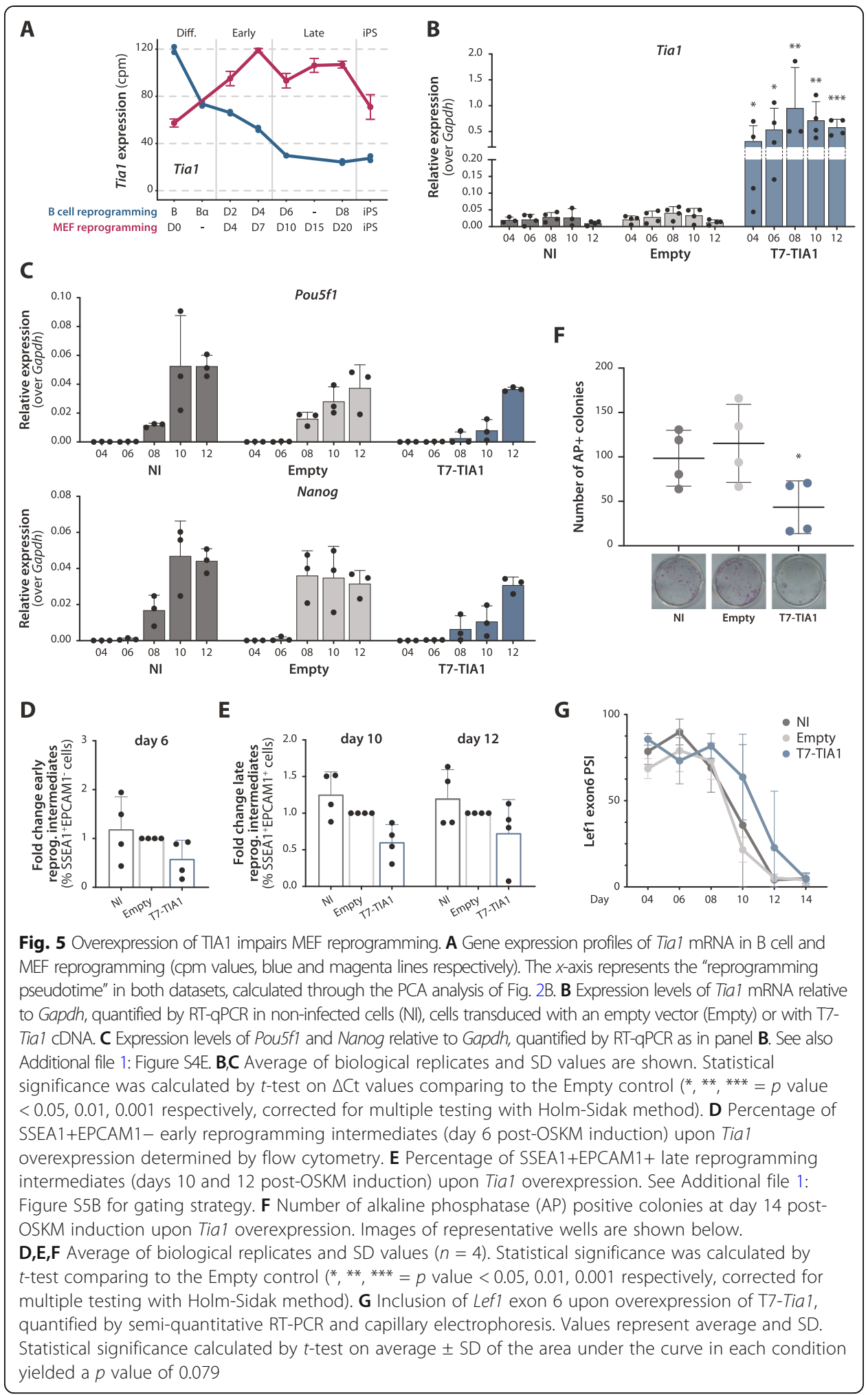

and UL1-dependent events were defined as those with $\mid \Delta \Delta \mathrm{PSI}$ (average shRNAs shSCR) $\mid \geq 10$ (only events selected by both specific shRNAs were considered, $54 \%$ and $60 \%$ overlap respectively, colored dots in Fig. 6C and Additional file 1: Figure S6C). For 


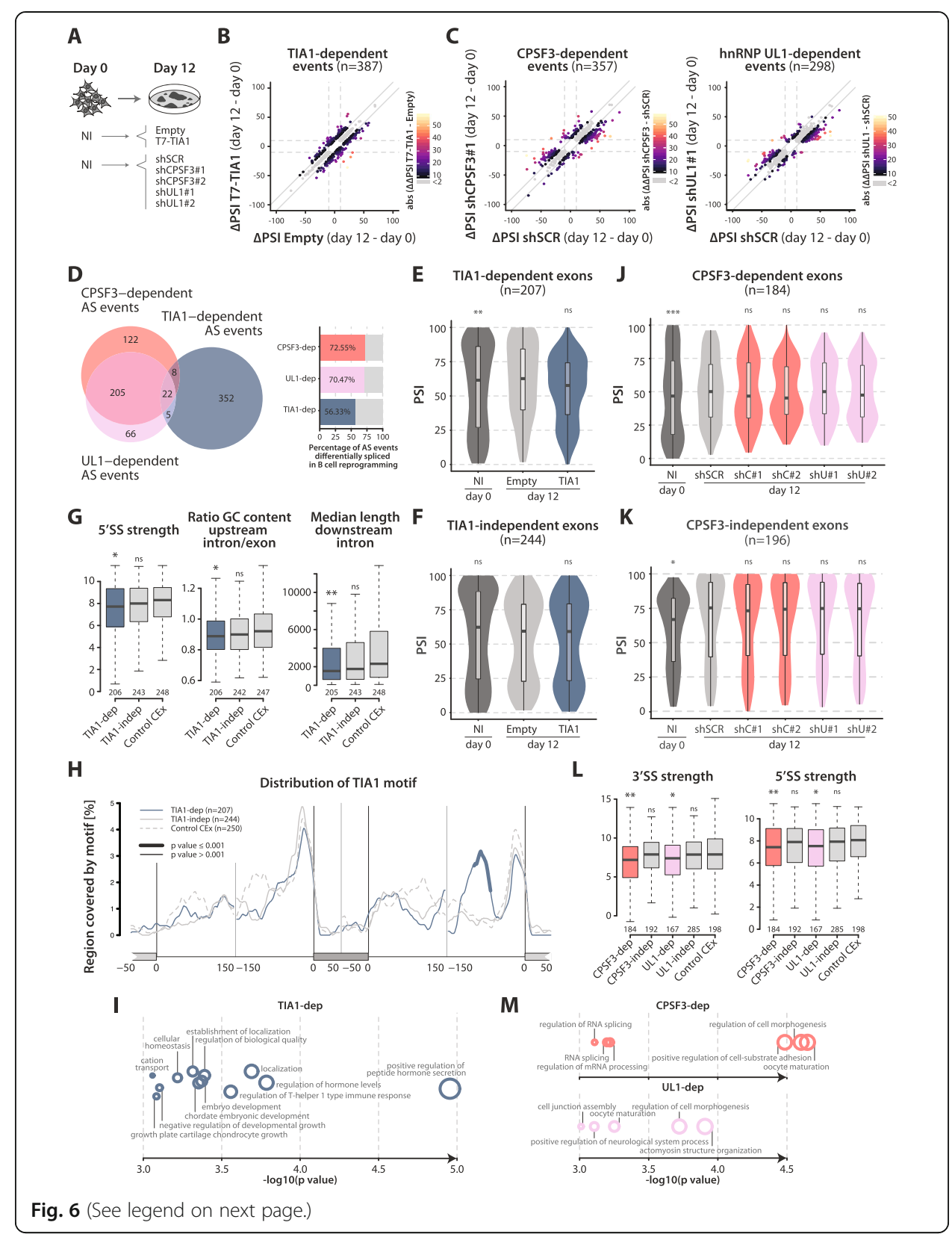


(See figure on previous page.)

Fig. 6 Knockdowns of CPSF3 or hnRNP UL1 and overexpression of TIA1 regulate AS during reprogramming. A Schematic representation of the experiments performed to study the effect of TIA1 overexpression / CPSF3 or hnRNP UL1 knockdown by RNA-seq. B TIA1-dependent events detected during reprogramming. The $x$-axis represents the $\triangle P S I$ value between Empty day 12 and day 0 . The $y$-axis represents the $\triangle P S I$ value between T7-TIA1 day 12 and day 0 control. TIA1-dependent events $(\mid \triangle \triangle P S I(T 7-T I A 1-$ Empty) $\mid \geq 10)$ are represented by colored dots (palette representing the $\mid \triangle \triangle P S I(T 7-T I A 1-$ Empty) $\mid$ value, $n=387$ ). TIA1independent events $(\mid \triangle \triangle P S I(T 7-T I A 1-$ Empty $) \mid<2)$ are represented by grey dots $(n=558)$. C CPSF3- and UL1-dependent events detected during reprogramming (left and right, respectively). The $x$-axis represents the $\triangle P S I$ value between shSCR day 12 and day 0 control. The $y$-axis represents the $\triangle P S I$ value between shCPSF3\#1 or shUL1\#1 day 12 and day 0 control. CPSF3/UL1-dependent events ( $\triangle P S I$ (average_shRNAs shSCR) $\geq 10$ ) are represented by non-grey-colored dots (palette representing the $\triangle \triangle P S I$ (average_shRNAs shSCR) value). CPSF3/UL1-independent events ( $\triangle P S I$ (average_shRNAs - shSCR) $<2$ ) are represented by grey dots. See Additional file 1: Figure S6C for $\triangle P S I$ values of the same events in shRNA\#2 conditions. D Venn diagram representing the overlap between CPSF3-, UL1-, and TIA1-dependent events (left, the number of events in each category is shown). Barplot representing the percentage of CPSF3-, UL1-, and TIA1-dependent events which are also differentially spliced in B cell reprogramming (right, the percentage of overlap in each category is indicated). $\mathbf{E}$ Violin plots representing the distribution of PSI values of TIA1dependent events in non-infected cells ( $\mathrm{NI}$, day 0 ) and day 12 cells infected with Empty or T7-Tial vectors. F Violin plots representing the distribution of PSI values of TIA1-independent events as in panel $\mathbf{E}$. E,F Statistical significance was calculated by Fisher's exact test comparing number of events with intermediate $(25<\mathrm{PSI}<75)$ or extreme PSI values (PSI $\geq 75$ or $\leq 25)$ in each condition against Empty control $\left({ }^{*},{ }^{*},{ }^{* * *}=p\right.$ value $<0.05,0.01,0.001$ respectively). See also Additional file 1: Figure S6D. G Boxplots representing the distribution of the indicated sequence features of TIA1-dependent exons, compared to TIA1-independent events and a random set of exons with intermediate PSI values not changing throughout reprogramming (Control CEx). Statistical significance was calculated using Matt, by paired Mann-Whitney $U$ test comparing each condition to the Control CEx set $\left(*^{*}, *^{*},{ }^{* *}=p\right.$ value $<0.05,0.01,0.001$ respectively). H RNA map representing the distribution of TIA1 binding motif in TIA1-dependent exons and flanking introns, compared to TIA1-independent and Control CEx. Thicker segments indicate regions in which enrichment of TIA1 motif is significantly different compared to Control CEx. I Gene Ontology (GO) terms enriched in genes containing TIA1-dependent events, compared to a background of all genes containing mapped AS events in the dataset. GO enrichment was calculated using GOrilla and GO terms were summarized for visualization using REVIGO. The $x$-axis and the size of each bubble represent the $-\log 10(p$ value) of each GO term. J Violin plots representing the distribution of PSI values of CPSF3-dependent events in non-infected cells (NI, day 0 ) and day 12 cells infected with shSCR or two shRNAs specific for CPSF3 (shC\#1 and shC\#2) or UL1 (shU\#1 and shU\#2). K Violin plots representing the distribution of PSI values of CPSF3-independent events as in panel $\mathbf{J}$. J,K Statistical significance was calculated by Fisher's exact test comparing number of events with intermediate $(25<\mathrm{PSI}<75)$ or extreme PSI values (PSI $\geq 75$ or $\leq 25)$ in each condition against shSCR control $\left(^{*},{ }^{* *},{ }^{* * *}=p\right.$ value $<0.05,0.01,0.001$ respectively). See also Additional file 1: Figure S6E. L Boxplots representing the distribution of sequence features of CPSF3- and UL1dependent exons, compared to the corresponding CPSF3- and UL1-independent events and Control CEX. Statistical significance was calculated using Matt, by paired Mann-Whitney $U$ test comparing each condition to the Control CEx set $\left({ }^{*},{ }^{* *},{ }^{* * *}=p\right.$ value $<0.05,0.01,0.001$ respectively). $\mathbf{M} \mathrm{GO}$ terms enriched in genes containing CPSF3- and UL1-dependent events, compared to a background of all genes containing mapped AS events in the dataset, performed as in panel I

each RBP, control events, changing during reprogramming but not affected by TIA1, CPSF3, or hnRNP UL1 manipulation, were classified as those differentially spliced between days 0 and 12, either in control or knockdown/overexpression conditions $(\mid \Delta \operatorname{PSI}($ day12 - day0) $\mid \geq 10$, range $\geq 5)$, that display minimal differences between the $\Delta$ PSI values in the two conditions (e.g., $\mid \Delta \Delta \operatorname{PSI}($ TIA1 - Empty) $\mid<2$ ) (grey dots in Fig. 6B, $\mathrm{C}$ and Additional file 1: Figure S6C). All sets are summarized in Additional file 5: Table S4.

We thus identified 387 TIA1-dependent events and 558 TIA1-independent events. Similarly, we identified 357 CPSF3-dependent and 298 UL1-dependent events (as well as 429 CPSF3-independent and 662 UL1-independent events). Interestingly, CPSF3dependent and UL1-dependent events showed a significant overlap (e.g., 70\% of UL1dependent events were also CPSF3-dependent events, of which 98\% changed in the same 
direction) whereas little overlap was observed with TIA1-dependent events (Fig. 6D, left panel). As we found no strong evidence for cross-regulation between CPSF3 and hnRNP UL1 factors (either in AS or in gene expression, Additional file 1: Figure S6B), these data suggest that CPSF3 and hnRNP UL1 contribute to a common program of AS changes relevant for cell reprogramming. Moreover, TIA1-, CPSF3-, and UL1-dependent events detected in MEFs showed high overlap with events that were also differentially spliced during B cell reprogramming, suggesting that these factors regulate AS events that generally contribute to the induction of pluripotency (Fig. 6D, right panel).

Focusing on cassette exon events, TIA1-dependent exons (but not TIA1-independent exons) displayed significantly higher PSI values at day 12 compared to day 0 of reprogramming, as well as a relative increase in exons displaying intermediate PSI values (Fig. 6E, F and Additional file 1: Figure S6E). These effects are altered by TIA1 overexpression (Fig. 6E), suggesting that in this system, the typical role of TIA1 is to repress exon inclusion.

Analysis of sequence features associated with TIA1-regulated exons performed using Matt [39] revealed weaker 5' splice sites, shorter median length of the flanking downstream introns and a larger difference in GC content between the alternative exons and their flanking upstream introns (Fig. 6G), suggestive of a strong dependence of these exons on the process of exon definition [40]. Notably, an enrichment in putative TIA1 binding motifs was detected about 100 nucleotides upstream of the distal 3' splice site (Fig. $6 \mathrm{H}$ ), suggesting that binding of TIA1 to this region might repress inclusion of the alternative exon (or enhance pairing between the distal splice sites), leading to exon skipping.

Gene Ontology (GO) enrichment analysis (GOrilla; [41]) on the set of genes containing TIA1-dependent AS events compared to a background list of all genes containing mapped AS events $(n=11132)$ showed an enrichment in functions related to peptide/ hormone secretion, T-helper cell functions, and embryonic development (Fig. 6I and Additional file 6: Table S5).

CPSF3- and UL1-dependent exons showed similar inclusion patterns: an increased proportion of intermediate PSIs was observed at day 12 in CPSF3- and UL1-dependent but not in CPSF3- and UL1-independent events (Fig. 6J, K and Additional file 1: Figure S6E). CPSF3- and UL1-dependent exons also showed higher PSI values at day 12 compared to day 0 , and an enrichment in exons displaying intermediate PSI values at the end of the reprogramming process (Fig. 6J, $\mathrm{K}$ and Additional file 1: Figure S6E). These effects were attenuated, specifically for CPSF3- and UL1-dependent exons, upon knockdown of either of these factors (Fig. 6J, K and Additional file 1: Figure S6E), supporting the notion that their regulatory programs overlap. Both CPSF3- and UL1-dependent exons displayed weaker $3^{\prime}$ and $5^{\prime}$ splice sites (Fig. 6L). GO analyses revealed that CPSF3- and UL1-dependent events belong to genes enriched in functional categories related to the regulation of cell morphogenesis, cell-substrate adhesion, and cytoskeleton organization (Fig. 6M and Additional file 6: Table S5). Changes in inclusion levels of selected CPSF3- and UL1-dependent events were validated by semi-quantitative RTPCR (Additional file 1: Figure S6F).

As CPSF3 was originally described as a cleavage / polyadenylation factor, we asked whether the CPSF3-regulated exons could be the result of changes in alternative polyadenylation (APA) sites. Not unexpectedly, quantification of poly-A usage performed 
with QAPA [42] revealed hundreds of genes with APA events affected by Cpsf3 knockdown during reprogramming (277 genes) and, interestingly, also many genes with APA events affected by Hnrnpul1 knockdown (237 genes) (| $\triangle \Delta$ PAU(shRNA-shSCR) $\geq 10$ ). However, the overlap between the genes / exons whose AS or APA were affected by Cpsf3 or Hnrnpul1 knockdown was very limited (Additional file 1: Figures S6G-H), suggesting that the mechanisms by which CPSF3 and hnRNP UL1 regulate alternative splicing and $3^{\prime}$ end processing are distinct and independent.

Taken together, these results suggest that the AS regulators TIA1, CPSF3, and hnRNP UL1 function in cell reprogramming through their activities on genes relevant for cell fate decisions. Moreover, CPSF3 and hnRNP UL1 act through a highly overlapping program of splicing changes, while TIA1 affects a different set of AS events. Distinct programs of AS and APA were associated with CPSF3 and hnRNP UL1.

\section{Discussion}

Somatic cell reprogramming holds great promise for fundamental research and medicine. In this study, we have characterized the dynamics of AS changes during the deterministic reprogramming of B cells into iPS cells and have identified three splicing regulators with a role in somatic cell reprogramming, as validated in perturbation experiments performed during MEF reprogramming.

The B cell reprogramming system is a two-step process, in which a pulse of the myeloid-specific transcription factor C/EBP $\alpha$ poises the cells for deterministic reprogramming by the subsequent induction of the OSKM factors, inducing early chromatin opening and epigenetic modifications that silence of the $\mathrm{B}$ cell-specific program and induce pluripotency [18-20,43]. While C/EBP $\alpha$ might not directly impact AS, it remains possible that the pulse of $\mathrm{C} / \mathrm{EBP} \alpha$ could induce changes in the expression of splicing regulatory factors (e.g., induction of CPSF3 or repression of hnRNP UL1 or TIA1) that trigger a program of post-transcriptional changes that contributes to cell reprogramming or that $\mathrm{C} /$ EBP $\alpha$ could have direct effects on the activity of splicing factors.

We observed widespread changes in AS, whose frequency increased as reprogramming progressed. Clustering analysis of cassette exons revealed groups of exons varying in inclusion at very early stages of $\mathrm{B}$ cell reprogramming (including following the $\mathrm{C} /$ EBP $\alpha$ pulse), but also at middle and late phases after OSKM induction. Of note, early clusters contain a larger proportion of exons predicted to disrupt open reading frames (ORFs) of isoforms predominantly expressed in the B cells, whereas middle clusters contain more ORF-preserving exons. Together with the observation that the majority of regulated introns tend to be retained (leading to ORF disruption) at early stages and are progressively spliced during reprogramming, these findings suggest that, in the transition from early to late stages, AS acts as a switch towards a gain in coding capacity, while at intermediate stages of reprogramming AS acts more commonly as a switch between isoforms.

The AS switch observed between day 4 and day 6 of B cell reprogramming coincides with a transcriptional switch, although the proportion of genes displaying both gene expression and AS changes is lower than expected (typically $1 / 3$ of alternatively spliced transcripts are controlled at the level of gene expression by nonsense-mediated decay [1, 26, 27, 44]. The transcriptomic changes observed during this transition might be mediated at least in part by the replacement of the culture medium from serum + LIF cell to N2B27-based 2i medium 
at day 6. Exons changing their inclusion levels during this of intermediate period displayed the highest overlap with exons differentially spliced in the reprogramming of MEFs, arguing for the general relevance of these changes in the induction of pluripotency.

Our analysis of changes in expression of RBPs during B cell reprogramming suggested potential regulators of AS, including factors already known to play a role in cell reprogramming such as the pluripotency repressors MBNL1/2 and CELF2 [7, 11], which are downregulated during $B$ cell reprogramming, and positive pluripotency regulators such as U2AF1 and hnRNP H1 [10, 14], which were upregulated during the process, consistent with results described for MEF reprogramming [17]. We focused on three candidate regulators of early AS changes, CPSF3, hnRNP UL1, and TIA1, because they could potentially contribute to the reprogramming advantage acquired by $\mathrm{B}$ cells poised by C/EBP $\alpha$ and/or to early events occurring during reprogramming. We found that perturbations of each of the three factors impaired the reprogramming of MEFs. We focused on functional tests in MEFs because of the more technical challenging two-step protocol required for reprogramming $B$ cells, the lower cellular yields, and the lower efficiency of transfection/infection of B cells, which greatly complicates knockdown and overexpression experiments. CPSF3 plays an important role in mRNA polyadenylation, recognizing the polyadenylation signal (PAS) together with the rest of the CPSF complex. In addition to the well-known intense crosstalk between last intron splicing and 3' end processing [31], CPSF2 and the CPSF complex have been shown to influence splicing of internal exons independently of cleavage and polyadenylation [28, $29,32]$. Furthermore, mutations of the CPSF3 yeast homolog Brr5/Ysh1 have been shown to strongly affect splicing, suggesting that CPSF3 could also play similar direct regulatory functions [33]. hnRNP UL1, instead, is a largely uncharacterized RBP known to participate in DNA damage response [45] and identified as a surface marker of human ES cells, although its functions in this context remain unknown [46]. Recent work in Zebrafish suggests a possible role for Hnrnpul1 in the regulation of AS [47].

Knockdown of Cpsf3 or Hnrnpul1 (with two independent shRNAs for each gene) caused a general repression or delay in MEF reprogramming, revealing their functions as promoters of somatic cell reprogramming. Sequence analysis of regulated AS events suggest that CPSF3 and hnRNP UL1 favor the definition of exons harboring relatively weak splice sites. Remarkably, the AS targets of CPSF3 and hnRNP UL1 identified by our RNA-seq analyses show a high overlap (without evidence of cross-regulation between the two proteins), suggesting a concerted mechanism of splice site selection, perhaps as components of the same complex. It is unlikely that the overlap is an indirect consequence of reduced/delayed reprogramming, because TIA1 overexpression also inhibits reprogramming and yet it is accompanied by largely non-overlapping changes in AS. Our results therefore suggest that multiple, separable AS programs contribute to the regulation of cell reprogramming and that these factors can contribute to reprogramming based upon their activities as splicing regulators, alternative polyadenylation regulators, or both.

TIA1 is a regulator of RNA metabolism influencing mRNA decay and AS implicated in cell proliferation, cell cycle progression, cellular stress, autophagy, and programmed cell death [35, 37, 38, 48] whose depletion induces mouse embryonic lethality [49].

We found that TIA1 overexpression at early stages of reprogramming represses the induction of pluripotency, as reported for MBNL proteins [7]. Binding of TIA1 to U- 
rich sequences downstream of weak 5'SS helps to recruit U1 snRNP and facilitates exon definition [35-37, 50], while it can also inhibit the inclusion of alternative exons located at a distance from its binding site [51]. As expected, TIA1-dependent exons are enriched in weak $5^{\prime}$ splice sites and other features that support a role in exon definition. Interestingly, TIA1-dependent exons in our system feature an enrichment of predicted U-rich TIA1 binding sites approximately 100 nucleotides upstream of the 3' splice site located in the downstream intron, suggesting that this configuration serves to repress the inclusion of exons that contribute to cell reprogramming. Since GO terms most enriched in genes bearing TIA1-dependent exons include "positive regulation of peptide hormone secretion," we hypothesize that TIA1-regulated transcripts could modulate functions related to the senescence-associated secretory phenotype (SASP), which was shown to promote reprogramming of somatic cells and dedifferentiation in cancer [52-54]. Indeed, 7 out of $23(30 \%)$ of the genes belonging to these GO terms were also found in a list of senescence- or SASP-associated genes (GeneCards database; [55]).

The finding that overexpression of TIA1 caused a delay in the conserved gradual skipping of Lef1 exon 6 observed during MEF reprogramming is consistent with a conserved role of the factor in reprogramming, as gradual skipping of this exon was also observed in middle stages of B cell reprogramming along with a decrease in expression of the gene. LEF1 is a transcription factor with important functions in embryonic and T cell development and activation [56-60]. As Lef1 exon 6 inclusion is promoted by CELF2 during $\mathrm{T}$ cell development and activation [61-63] and Celf2 expression rapidly decreases in both $\mathrm{B}$ cell and MEF reprogramming, CELF2 may participate-along with TIA1-in Lef1 exon 6 regulation during reprogramming. We observed that overexpression of Lef1 at the start of MEF reprogramming improved reprogramming efficiency (observed as an increase of pluripotency markers and percentage of reprogramming intermediates), with overexpression of the iPS-associated Lef1 exon 6-skipping isoform displaying stronger effects than the exon 6-including isoform, suggesting a functional role for this switch in cell reprogramming (Additional file 1: Figures S5D-K). We speculate that the gradual skipping of exon 6 affects the interaction of LEF1 with protein partners that either act as coactivators-e.g., ALY [64]—or corepressors-e.g., Groucho/TLE [65], differentially affecting the transcription of target genes. For example, the LEF1-inclusion isoform could promote cell proliferation and maintenance of B cell identity-as observed in the $\mathrm{C} / \mathrm{EBP} \alpha$-dependent transdifferentiation system and in pancreatic cancer cell lines [66,67], while gradual skipping of exon 6 might alter its effect on its target genes with the consequence of inducing pluripotency, most probably in a $\beta$ catenin-independent way.

\section{Conclusions}

Our results provide a comparison between AS changes occurring in two very different reprogramming systems and a wealth of information relevant for cell fate decisions and transitions to pluripotency. Furthermore, they demonstrate the functional involvement of the splicing regulators CPSF3, hnRNP UL1, and TIA1 as activators or repressors of efficient cell reprogramming. Our work significantly extends previous knowledge on RNA processing during reprogramming and suggests that AS changes play functional 
roles during the entire transition of somatic into pluripotent stem cells. It would be interesting to determine whether the AS/APA changes and regulators identified in our work play similar roles during the specification of pluripotent stem cells in early embryo development.

\section{Methods}

\section{RNA sequencing}

For RNA-seq of B cell reprogramming and MEF reprogramming upon TIA1 overexpression and knockdown of CPSF3 and hnRNP UL1, stranded libraries were prepared from samples and sequenced on an Illumina HiSeq 2500 using a 2x125nt paired-end protocol (see "Availability of data and materials"). Duplicates were sequenced for each condition, with samples pooled in separate lanes. RNA-seq data (triplicates of 2x100nt paired-end sequencing) of MEF reprogramming was downloaded from GEO Database ([68], see "Availability of data and materials").

\section{Gene expression and alternative splicing analyses}

Reads mapping (mm10 annotation) was performed with STAR v2.7.1a [69] and gene expression analysis was performed using the edgeR package v3.16.5 [25, 70]. A minimum of 5 counts per million (cpm) was required in 33\% of both datasets ( 5 samples for B cell reprogramming, 6 samples for MEF reprogramming) and a minimum coefficient of variation of 0.2 was required.

Alternative splicing (AS) analysis was performed using vast-tools software v2.2.2 [21, 71]. Strand-specific mapping was performed and only events with a minimum of 10 actual reads per sample were considered (VLOW quality score). PSI values for single replicates were quantified for all types of alternative events, including simple and complex cassette exons (S, C1, C2, C3), microexons (MIC), alternative $5^{\prime}$ and $3^{\prime}$ splice sites (Alt5, Alt3) and retained introns (IR-S, IR-C). For cassette exon events (referred to as CEx), PSI values of all annotated exons were also quantified with vast-tools (Annotation module ANN). For both B cell and MEF reprogramming, all possible pairwise comparisons between samples were performed, selecting differentially spliced events with a $|\Delta \mathrm{PSI}| \geq 10$ and a minimum range of 5 between samples.

For TIA1 overexpression experiments, TIA1-dependent events were defined as events with $|\Delta \Delta \mathrm{PSI}| \geq 10$, where $\Delta \Delta \mathrm{PSI}=(\Delta \mathrm{PSI}$ TIA1_day12 - NI_day 0$)-(\Delta \mathrm{PSI}$ Empty day12 - NI_day0). TIA1-independent events were instead defined as events changing in any of the two conditions $(|\Delta \mathrm{PSI}|$ TIA1_day12 - NI_day0 $\geq 10)$ or $(|\Delta \mathrm{PSI}|$ Empty_ day12 - NI_day0 $\geq 10)$ and having minimal difference between the two conditions $(|\Delta \Delta \mathrm{PSI}|<2)$.

Similarly, CPSF3- or UL1-dependent events were events for which in both shRNAs $|\Delta \Delta \mathrm{PSI}| \geq 10$, where $\Delta \Delta \mathrm{PSI}=(\Delta \mathrm{PSI}$ average_shC/U_day12 - NI_day0 $)-(\Delta \mathrm{PSI}$ shSCR day12 - NI_day0). CPSF3- or UL1-independent events were instead defined as events changing in any of the knockdown or control conditions $(|\Delta \mathrm{PSI}|$ average_shC/U_day12 - NI_day0) or $(|\Delta \mathrm{PSI}|$ shSCR_day12 - NI_day0 $\geq 10)$ and having minimal difference between the two conditions $(|\Delta \Delta \mathrm{PSI}|<2)$. 


\section{Clustering analysis of alternatively spliced exons and correlation of gene expression profiles}

PSI values of exons differentially spliced in at least one pair of B cell reprogramming samples $(n=4556)$ were scaled and centered (referred to as "scaled PSI" values). Fuzzy $c$-means clustering analysis was carried out using the R package $M f u z z[22,72]$ on the scaled PSI values. The optimal number of clusters (12) was selected on the basis of the minimum distance to the cluster centroid. A membership value was assigned to correlate gene expression profiles to each AS clusters, either of genes containing the AS exons of each cluster or of genes encoding RBPs. To each scaled cpm values vector, we attributed a membership value to the centroid of each cluster or to its negative.

\section{Correlation of $\mathrm{B}$ cell reprogramming stages}

Pearson correlation coefficient for B cell reprogramming stages was calculated on the most variable expressed genes $(\mathrm{cpm} \geq 5$ in at least 5 samples and coefficient of variation $\geq 0.73864$, corresponding to the 3rd quartile, $n=2961$ ) or the most variable exons (differentially spliced in at least one pairwise comparison and coefficient of variation $\geq 0.4107$, corresponding to the 3rd quartile, $n=1139$ ).

\section{Prediction of the protein impact of alternative exons}

Alternative exons detected by vast-tools were classified as described in vastDB v2.2.2 [21]. Briefly, following division according to their location in non-coding RNAs, untranslated regions ( $5^{\prime}$ or $3^{\prime}$ UTRs) or open reading frame (ORF), exons were predicted to disrupt the ORF if their inclusion or skipping would induce a frameshift in their ORF or if they would induce a premature stop codon (PTC) predicted to be targeted by nonsense-mediated decay (NMD) or truncating the protein by more than 300 amino acids. The rest of the ORF-mapping events were predicted to preserve the transcript coding potential.

\section{Principal component analysis (PCA) and comparison between B cell and MEF reprogramming}

Gene expression values were filtered for minimum variation (coefficient of variation $\geq$ 0.66 , corresponding to the 3rd quartile, $n=2679$ ), scaled and centered (referred to as "scaled cpm" values). Principal component analysis (PCA) was performed on scaled cpm values of the most variable genes expressed in both B cell and MEF reprogramming. The groups shown were obtained by $k$-means clustering. For heatmaps representing scaled PSI and scaled cpm values in B cell and MEF reprogramming, hierarchical clustering was performed on values of both datasets using Ward's method and Euclidean distance as the distance metric.

\section{Correlation of RNA-binding proteins expression to AS clusters}

The lists of Mus musculus RNA-binding proteins (RBPs) and splicing-related proteins were downloaded from the Uniprot database. For RBPs, Uniprot keywords had to match "RNA-binding [KW-0694]," "mRNA splicing [KW-0508]," "mRNA processing [KW-0507]," or "Spliceosome [KW-0747]." Splicing-related RBPs were defined if keywords were matching either "mRNA splicing [KW-0508]" or "Spliceosome [KW- 0747]." 
Filtered gene expression values were scaled as described above and a membership value was attributed to each RBP profiles for each AS cluster centroid and to its negative (RBPs with membership $\geq 0.3$ are shown).

\section{Sequence feature analysis and RNA maps}

Sequence features of exons and flanking introns were analyzed using Matt software v1.3.0 [39]. Features of TIA1-, CPSF3-, or UL1-dependent exons were compared with the corresponding independent exons and with a set of control cassette exons, representing alternative exons not changing during reprogramming. Specifically, the union of alternative non-changing exons (AS_NC sets, $10<$ average PSI $<90$ and $\Delta \mathrm{PSI} \leq 2$ ) in all conditions of each dataset was generated, TIA1- or CPSF3/UL1-dependent events were excluded and a random set was selected, with a size similar to TIA1- or CPSF3-/ UL1-dependent and TIA1- or CPSF3-/UL1-independent exon sets. Maximum entropy score is calculated as an approximation of splice site strength [73]. RNA maps for TIA1 M075 motif [74] were generated for the first and last $50 \mathrm{nt}$ of exons and the first and last $150 \mathrm{nt}$ of introns (sliding window $=31, p$ value $\leq 0.05$ with 1000 permutations).

\section{Statistical analyses and plots}

Statistical tests were performed as indicated in figures legends with R (v3.6.1) or GraphPad Prism (version 8). Heatmaps were plotted with ggplot2 package or heatmap.2 function. Venn diagrams were generated with VennDiagram package [75].

\section{Gene Ontology enrichment analysis}

Genes bearing the differentially spliced exons belonging to each set were analyzed for GO term enrichment in contrast to all genes containing any mapped AS event. Analysis was carried out with the "two unranked lists of genes" module of GOrilla (Gene Ontology enRIchment anaLysis and visuaLizAtion) tool [41] and summarized using REViGO [76]. Statistical significance was defined with $p$ value $<1 \mathrm{e}-03$. Only enriched terms for GO process are shown.

\section{Alternative polyadenylation analysis}

Transcript quantification was performed with Salmon v1.1.0 [77]. QAPA v1.3.0 was used to quantify poly-A site usage for each sample of the knockdown reprogramming dataset, based on the combined annotation of GENCODE and PolyAsite databases as described in [42]. A value of poly-A usage (PAU) was obtained for each site and filtering was applied as described in [42]: transcripts were selected for minimum expression ( 3 transcripts per million (tpm) in at least 10 out of 12 samples) and for number of poly-A sites (at least 2). Similarly to what was performed for AS, CPSF3- or UL1-dependent APA events were poly-A sites for which in both shRNAs $|\triangle \triangle \mathrm{PAU}| \geq 10$, where $\triangle \triangle \mathrm{PAU}=(\triangle \mathrm{PAU}$ shC/U\#1/2_day12 - NI_day0 $)-$ ( $\triangle$ PAU shSCR_day12 - NI_day0). Overlaps between the coordinates of AS events (largest junction) and APA events (last exon coordinates) were calculated with Bedtools v2.25.0 [78]. 


\section{Cell culture}

Platinum E cells (PlatE), 293 T/17 cells, stromal S17 cells, and primary MEFs serving as feeder cells for B cell reprogramming were kindly provided by the group of $\mathrm{T}$. Graf and cultured in Glutamax Dulbecco's modified Eagle's medium (DMEM, Life Technologies) supplemented with $10 \%$ fetal bovine serum (FBS) and penicillin/streptomycin antibiotics (PenStrep, $50 \mathrm{U} / \mathrm{ml}$ penicillin; $50 \mu \mathrm{g} / \mathrm{ml}$ streptomycin). All cells were maintained at $37{ }^{\circ} \mathrm{C}$ under $5 \% \mathrm{CO}_{2}$ and routinely tested for mycoplasma.

\section{Lentivirus production}

$293 \mathrm{~T} / 17$ cells were seeded on gelatin-coated $10-\mathrm{cm}$ plates at a density of $3 \times 10^{6}$ cells/ plate and incubated overnight. The following day, medium was replaced approximately $2 \mathrm{~h}$ before transfection. For each $10-\mathrm{cm}$ plate, $10 \mu \mathrm{g}$ of the plasmid of interest, $2.5 \mu \mathrm{g}$ of VSV-G, and $7.5 \mu \mathrm{g}$ of $\mathrm{p} \Delta 8.9$ were mixed with $61 \mu \mathrm{l}$ of $\mathrm{CaCl} 22.5 \mathrm{M}$ (Sigma) and endotoxin-free water up to $500 \mu \mathrm{l}$. While bubbling, $500 \mu \mathrm{l}$ of $2 \times \mathrm{HBS}$ pH $7.2(281 \mathrm{mM}$ $\mathrm{NaCl}, 100 \mathrm{mM}$ HEPES, $1.5 \mathrm{mM} \mathrm{Na} 2 \mathrm{HPO} 4)$ were added dropwise, and the mix was incubated for $10 \mathrm{~min}$ at room temperature (RT). The solution was added dropwise to the $293 \mathrm{~T} / 17$ cells, followed by incubation for $24 \mathrm{~h}$ at $37^{\circ} \mathrm{C}$. The following day, medium was substituted with $6 \mathrm{ml}$ of fresh medium (reprogramming medium for MEF reprogramming). Viral medium was collected 48 and $72 \mathrm{~h}$ after transfection, filtered $(0.22 \mu \mathrm{m}$ pore size), and supplemented as needed.

\section{Retrovirus production}

$4.5 \times 10^{6}$ PlatE cells were seeded in gelatin-coated $10-\mathrm{cm}$ plates $(0.1 \%$ gelatin, Millipore) the day before transfection. To improve efficiency, chloroquine was added approximately $1 \mathrm{~h}$ before transfection to a final concentration of $30 \mu \mathrm{M}$. For each plate, $20 \mu \mathrm{g}$ of plasmid was mixed with $60 \mu \mathrm{l}$ of $\mathrm{CaCl} 22.5 \mathrm{M}$ (Sigma) and endotoxin-free water up to $500 \mu \mathrm{l}$. While bubbling, $500 \mu \mathrm{l}$ of $2 \times \mathrm{HBS}$ pH $7.2(281 \mathrm{mM} \mathrm{NaCl}, 100 \mathrm{mM}$ HEPES, $1.5 \mathrm{mM}$ Na2HPO4) were added dropwise, and the mix was incubated for 10 min at room temperature (RT). The solution was added dropwise to the PlatE cells and transfected cells were incubated for 8-10 h. Medium was substituted with $6 \mathrm{ml}$ of fresh medium (reprogramming medium for MEF reprogramming). Viral medium was collected the day after transfection and the following one, filtered $(0.22 \mu \mathrm{m}$ filter pore size), and supplemented as needed.

\section{C/EBPa-dependent $\mathrm{B}$ cell reprogramming}

Primary B cell reprogramming was performed as described in [18-20, 79].

\section{MEF reprogramming}

Primary MEFs (P0), obtained from male embryos of a Collagen-OKSM, M2rtta ${ }^{+}$, mouse line [30] were cultured on gelatin-coated plates in MEF medium (DMEM; high glucose + Glutamax, FBS 10\%, PenStrep 1×, Sodium Pyruvate $1 \mathrm{mM}$, HEPES $30 \mathrm{mM}$, NEAA 1x, $\beta$-mercaptoethanol $0.1 \mathrm{mM}$ ). Primary MEFs were expanded for a maximum of 2 passages before inducing reprogramming. Early passage MEFs were plated in MEF medium on gelatin-coated 6-well plates at a density of 70,000 cells/ well. The following day, infection was performed by substituting the medium with 
the filtered retroviral or lentiviral supernatant (prepared in reprogramming medium), supplemented with LIF and doxycycline to induce reprogramming. Two subsequent infections were performed, $12 \mathrm{~h}$ apart, after which fresh reprogramming medium was added. The following day (day 2), cells were trypsinized and FACSsorted for GFP expression by flow cytometry. Sorted MEFs were seeded (10,000 cells/well of 12-well plate) on irradiated feeders on gelatin-coated plates (plated the previous day 100,000 cells/well of 12-well plate). Medium was substituted every 2 days starting from day 4. Harvesting was performed every 2 days with trypsin $0.25 \%$ to ensure the complete dissociation of the feeder layer. Doxycycline was withdrawn from the culture at day 12 post-OSKM induction and AP staining was performed at day 14 with the Alkaline Phosphatase Kit II (StemGent), according to the manufacturer's instructions. The number of $\mathrm{AP}^{+}$colonies was quantified with ImageJ software (colony size between 20 and 2000 pixels).

Reprogramming medium is composed by DMEM, high glucose + Glutamax, ESqualified FBS 15\%, PenStrep 0.5×, Sodium Pyruvate $1 \mathrm{mM}$, HEPES $30 \mathrm{mM}$, NEAA $1 \times$, and $\beta$-mercaptoethanol $0.1 \mathrm{mM}$. LIF $(1000 \mathrm{U} / \mathrm{ml})$ and doxycycline $(1 \mu \mathrm{g} / \mathrm{ml})$ were added freshly.

\section{Flow cytometry analysis}

Cells were trypsinized and stained with a mix of antibodies SSEA1-eFluor 660 (MC480, eBioscience) and EpCAM-PE (G8.8, eBioscience), both at a 1:50 dilution in $100 \mu \mathrm{l}$ per sample. Staining was carried out by incubating on ice for $20 \mathrm{~min}$, followed by washing and staining with DAPI (Sigma). Flow cytometry analysis was performed with a BD LSR II analyzer. Gates and compensation between FITC and PE were adjusted on nonstained controls. In total, 10,000 events (alive cells) per sample were acquired.

\section{Cloning of LEF1 isoforms and TIA1 in retroviral vectors}

Lef1 isoforms were amplified from bulk cDNAs of B cells and iPS (RNA-seq samples) and cloned using Gibson technology [80] into a MIG-pMSCV retroviral vector including an IRES-GFP element. Kozak consensus sequences and an N-terminal T7 tag were inserted before the LEF1 ORF to ensure efficient translation and discrimination from endogenous Lef1.

cDNA of mouse Tia1 was purchased from GenScript in pcDNA3.1 vectors (clone ID: $\mathrm{OMu} 08423 \mathrm{D}$ ) and cloned using a similar Gibson strategy into the MIG-pMSCV retroviral vector bearing the N-terminal T7 and IRES-GFP elements. Gibson reaction master mixes were provided by the CRG Protein Technologies Core Facility.

\section{Short hairpin RNAs}

Five MISSION shRNAs specific for each splicing regulator were purchased from Sigma in pLKO lentiviral vectors, and their effects were compared with those of SHC002 mammalian non-targeting MISSION shRNA (Sigma). The effect of each shRNA was tested on MEFs and E14 embryonic stem cells, and knockdown efficiency was assessed by RT-qPCR at $72 / 96 \mathrm{~h}$ post-infection using primers specific for each target and normalized by the expression of two housekeeping genes 
(Gapdh and Rplp0). The two shRNAs displaying the largest knockdown effects in both cell lines were selected (sequences shown in Additional file 7: Table S6).

RNA extraction and retrotranscription, semi-quantitative RT-PCR, and real-time qPCR (RT-qPCR)

RNA extraction and DNAse treatment was performed using Maxwell simplyRNA kit (Promega) or RNeasy Mini kit (Qiagen), following the manufacturers' instructions. In total, $200 \mathrm{ng}$ of total RNA were reverse-transcribed with Superscript III (Invitrogen) following the manufacturer's recommendations.

PCR reactions were carried out using GoTaq enzyme (Promega) with $1 \mu \mathrm{l}$ of cDNA diluted 1:5. To quantify inclusion of alternatively spliced exons, capillary electrophoresis was performed using a Labchip GX Caliper workstation (Caliper, Perkin Elmer) at the CRG Protein Technologies Core Facility. The nanomolar content of each band was extracted with LabChip GX software and PSI values were calculated as the ratio between the inclusion amplicon and the sum of inclusion and skipping amplicons.

Real-time quantitative PCR (RT-qPCR) was performed on a ViiA7 Real Time PCR System (Applied Biosystems). Reactions in a total volume of $10 \mu$ l contained $2 \times$ SYBR Green Master Mix (Applied Biosystems), primers $400 \mathrm{nM}$ and $1 \mu \mathrm{l}$ of previously synthetized cDNA, diluted 1:5-20. The output $\mathrm{Ct}$ values were normalized by the expression of the housekeeping gene Gapdh (unless differently stated) and analyzed with $\Delta \mathrm{Ct} /$ $\Delta \Delta \mathrm{Ct}$ method. All primers sequences are listed in Additional file 7: Table S6.

\section{Supplementary Information}

The online version contains supplementary material available at https:/doi.org/10.1186/s13059-021-02372-5.

Additional file 1. Supplementary figures and corresponding legends.

Additional file 2: Table S1. Cassette exon events differentially spliced in B cell reprogramming. This table includes the information about the AS cluster each exon belongs to and the predicted effect on its transcript. Data for other types of events is available upon request.

Additional file 3: Table S2. Cassette exon events differentially spliced in MEF reprogramming. This table includes the information about the overlap with B cell reprogramming. Data for other types of events is available upon request.

Additional file 4: Table S3. Gene expression levels of RBPs correlating with AS clusters (positive and negative predicted regulators).

Additional file 5: Table S4. CPSF3-, UL1- and TIA1-dependent and-independent events. This table includes the sets of control cassette exons (CEX) used for KD (CPSF3- and UL1-dependent CEX) or OE (TIA1-dependent CEx).

Additional file 6: Table S5. Gene Ontology terms enriched in CPSF3-, UL1- and TIA1-dependent events. This table includes outputs from GOrilla and REViGO analyses.

Additional file 7: Table S6. Sequences of primers and shRNAs used in this study.

Additional file 8. Review history.

\section{Acknowledgements}

We thank the CRG Genomics Unit for sequencing; the CRG Tissue Engineering Unit for the inactivated MEF feeders: the CRG/UPF FACS Unit for FACS sorting; Daniel Soronellas for help with the Mfuzz package and Konrad Hochedlinger for the Col-OKSM reprogrammable mouse strain. We thank members of Manuel Irimia's, T.G.'s and J.V.'s groups for discussions; Manuel Irimia and members of J.V.'s group for critical reading of the manuscript.

Review history

The review history is available as Additional file 8. 


\section{Authors' contributions}

C.V., T.G., and J.V. conceived the study. C.V., P.P., J.L.S., and J.V. designed experiments and data analyses. C.V. and J.V. wrote the manuscript with input from all authors. C.V. performed the majority of the experimental work and data analyses (RNA-seq analyses, MEF reprogramming, and molecular biology experiments). R.S., B.D.S., and C.B. performed the B cell reprogramming experiments for RNA-seq. A.R.R. performed some RT-PCR validations and provided technical support. S.G, A.M. and B.P. provided reprogrammable MEFs. J.V. and T.G. acquired funding. The authors read and approved the final manuscript.

\section{Authors' information}

Twitter handles: @clau_vivori (Claudia Vivori); @R_Stadhouders (Ralph Stadhouders); @AnnaRR82 (Anna Ribó Rubio); @AnMallol (Anna Mallol); @Payerlab (Bernhard Payer); @JuanValcarcel5 (Juan Valcárcel).

\section{Funding}

C.V. was recipient of an FPI-Severo Ochoa Fellowship from the Spanish Ministry of Economy and Competitiveness. Work in J.V. laboratory is supported by the European Research Council (ERC AdvG 670146), AGAUR, Spanish Ministry of Economy and Competitiveness (BFU 2017 89308-P) and the Centre of Excellence Severo Ochoa. Work in T.G.'s laboratory was supported by the European Research Council FP7/2007-2013 (ERC Synergy Grant 4D-Genome) the Ministerio de Educación y Ciencia (SAF.2012-37167) and AGAUR. We acknowledge support of the Spanish Ministry of Science and Innovation to the EMBL partnership and the CERCA Programme / Generalitat de Catalunya.

\section{Availability of data and materials}

RNA-seq data of MEF reprogramming upon TIA1 overexpression and knockdown of CPSF3 and hnRNP UL1, generated during the current study, have been deposited in NCBI's Gene Expression Omnibus [68] and are accessible through GEO Series accession number GSE158633 [81]. The code used to process and analyze these data was released under the GNU GPL-3 license (https://doi.org/10.5281/zenodo.4724548) and is available at https://github.com/cvivori/ASreprogramming-KD-OE [82]. The RNA-seq data of B cell [20] and MEF reprogramming [17] analyzed in the current study are available under GEO Series accession numbers GSE96611 and GSE70022, respectively. The code used to process and analyze these data was released under the GNU GPL-3 license (https://doi.org/10.5281/zenodo.4723460) and is available at https://github.com/cvivori/AS-BandMEF-reprogramming [83].

\section{Declarations}

\section{Ethics approval and consent to participate}

Not applicable.

\section{Competing interests}

The authors declare no competing interests.

\section{Author details}

${ }^{1}$ Centre for Genomic Regulation (CRG), The Barcelona Institute of Science and Technology, Carrer del Dr. Aiguader 88, 08003 Barcelona, Spain. ${ }^{2}$ Universitat Pompeu Fabra (UPF), Carrer del Dr. Aiguader 88, 08003 Barcelona, Spain. ${ }^{3}$ Present address: The Francis Crick Institute, 1 Midland Road, London NW1 1AT, UK. ${ }^{4}$ Present address: Friedrich Miescher Institute for Biomedical Research, Maulbeerstrasse 66/Swiss Institute of Bioinformatics, 4058 Basel, Switzerland. ${ }^{5}$ Present address: Departments of Pulmonary Medicine and Cell Biology, Erasmus MC, Rotterdam, The Netherlands. ${ }^{6}$ Present address: Department of Molecular and Cellular Biology, Baylor College of Medicine, One Baylor Plaza, Alkek Bldg Room

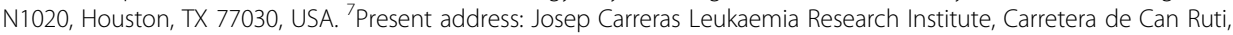
Camí de les Escoles, s/n, 08916 Badalona, Spain. ${ }^{8}$ Institució Catalana de Recerca i Estudis Avançats (ICREA), Passeig Lluís Companys 23, 08010 Barcelona, Spain.

Received: 14 December 2020 Accepted: 5 May 2021

Published online: 03 June 2021

\section{References}

1. Pan Q, Shai O, Lee LJ, Frey BJ, Blencowe BJ. Deep surveying of alternative splicing complexity in the human transcriptome by high-throughput sequencing. Nat Genet. 2008;40(12):1413-5. Available from: http://www.nature.com/a rticles/ng.259. https://doi.org/10.1038/ng.259.

2. Wang ET, Sandberg R, Luo S, Khrebtukova I, Zhang L, Mayr C, et al. Alternative isoform regulation in human tissue transcriptomes. Nature. 2008;456(7221):470-6. https://doi.org/10.1038/nature07509.

3. Baralle FE, Giudice J. Alternative splicing as a regulator of development and tissue identity. Nat Rev Mol Cell Biol. 2017; 18(7):437-51. Available from: http://www.nature.com/articles/nrm.2017.27. https://doi.org/10.1038/nrm.2017.27.

4. Yeo GW, Coufal NG, Liang TY, Peng GE, Fu XD, Gage FH. An RNA code for the FOX2 splicing regulator revealed by mapping RNA-protein interactions in stem cells. Nat Struct Mol Biol. 2009;16(2):130-7. Available from: http://www.na ture.com/articles/nsmb.1545. https://doi.org/10.1038/nsmb.1545.

5. Salomonis N, Schlieve CR, Pereira L, Wahlquist C, Colas A, Zambon AC, et al. Alternative splicing regulates mouse embryonic stem cell pluripotency and differentiation. Proc Natl Acad Sci U S A. 2010;107(23):10514-9 Available from: http://www.pnas.org/cgi/doi/10.1073/pnas.0912260107.

6. Gabut M, Samavarchi-Tehrani P, Wang X, Slobodeniuc V, O'Hanlon D, Sung HK, et al. An alternative splicing switch regulates embryonic stem cell pluripotency and reprogramming. Cell. 2011;147(1):132-46 Available from: https://doi. org/10.1016/j.cell.2011.08.023. 
7. Han H, Irimia M, Ross PJ, Sung HK, Alipanahi B, David L, et al. MBNL proteins repress ES-cell-specific alternative splicing and reprogramming. Nature. 2013;498(7453):241-5. Available from: http://www.nature.com/articles/nature12270. https:// doi.org/10.1038/nature12270

8. Venables JP, Lapasset L, Gadea G, Fort P, Klinck R, Irimia M, et al. MBNL1 and RBFOX2 cooperate to establish a splicing programme involved in pluripotent stem cell differentiation. Nat Commun. 2013;4(1):2480 Available from: http://www.na ture.com/articles/ncomms3480.

9. Lu Y, Loh YH, Li H, Cesana M, Ficarro SB, Parikh JR, et al. Alternative splicing of MBD2 supports self-renewal in human pluripotent stem cells. Cell Stem Cell. 2014;15(1):92-101. Available from: http://linkinghub.elsevier.com/retrieve/pii/S1934 590914001398. https://doi.org/10.1016/j.stem.2014.04.002.

10. Yamazaki T, Liu L, Lazarev D, Al-Zain A, Fomin V, Yeung PL, et al. TCF3 alternative splicing controlled by hnRNP H/F regulates E-cadherin expression and hESC pluripotency. Genes Dev. 2018;32(17-18):1161-74 Available from: http:// genesdev.cshlp.org/lookup/doi/10.1101/gad.316984.118.

11. Solana J, Irimia M, Ayoub S, Orejuela MR, Zywitza V, Jens M, et al. Conserved functional antagonism of CELF and MBNL proteins controls stem cell-specific alternative splicing in planarians. Elife. 2016;5(AUGUST):1-29 Available from: https:// elifesciences.org/articles/16797.

12. Takahashi K, Yamanaka S. Induction of pluripotent stem cells from mouse embryonic and adult fibroblast cultures by defined factors. Cell. 2006;126(4):663-76. Available from: https://inkinghub.elsevier.com/retrieve/pii/\$0092867406009767. https://doi.org/10.1016/j.cell.2006.07.024.

13. Toh CXD, Chan JW, Chong ZS, Wang HF, Guo HC, Satapathy S, et al. RNAi reveals phase-specific global regulators of human somatic cell reprogramming. Cell Rep. 2016;15(12):2597-607. Available from: http://linkinghub.elsevier.com/ retrieve/pii/S2211124716306477. https://doi.org/10.1016/j.celrep.2016.05.049.

14. Ohta S, Nishida E, Yamanaka S, Yamamoto T. Global splicing pattern reversion during somatic cell reprogramming. Cell Rep. 2013;5(2):357-66. Available from: https://linkinghub.elsevier.com/retrieve/pii/S2211124713005391. https://doi.org/1 0.1016/j.celrep.2013.09.016.

15. Zavolan M, Kanitz A. RNA splicing and its connection with other regulatory layers in somatic cell reprogramming. Curr Opin Cell Biol. 2018;52:8-13. Available from: http://inkinghub.elsevier.com/retrieve/pii/S095506741730162X. https://doi. org/10.1016/j.ceb.2017.12.002.

16. Kanitz A, Syed AP, Kaji K, Zavolan M. Conserved regulation of RNA processing in somatic cell reprogramming. BMC Genomics. 2019;20(1):100 Available from: https://bmcgenomics.biomedcentral.com/articles/10.1186/s12864-01 9-5438-2.

17. Cieply B, Park JW, Nakauka-Ddamba A, Bebee TW, Guo Y, Shang X, et al. Multiphasic and dynamic changes in alternative splicing during induction of pluripotency are coordinated by numerous RNA-binding proteins. Cell Rep. 2016;15(2):247-55. Available from: https://linkinghub.elsevier.com/retrieve/pii/S2211124716302819. https://doi. org/10.1016/j.celrep.2016.03.025.

18. Di Stefano B, Sardina JL, Van Oevelen C, Collombet S, Kallin EM, Vicent GP, et al. C/EBPa poises B cells for rapid reprogramming into induced pluripotent stem cells. Nature. 2014;506(7487):235-9. Available from: http://www.nature. com/articles/nature12885. https://doi.org/10.1038/nature12885.

19. Di Stefano B, Collombet S, Jakobsen JS, Wierer M, Sardina JL, Lackner A, et al. C/EBPa creates elite cells for iPSC reprogramming by upregulating Klf4 and increasing the levels of Lsd1 and Brd4. Nat Cell Biol. 2016;18(4):371-81. Available from: http://www.nature.com/articles/ncb3326. https://doi.org/10.1038/ncb3326.

20. Stadhouders R, Vidal E, Serra F, Di Stefano B, Le Dily F, Quilez J, et al. Transcription factors orchestrate dynamic interplay between genome topology and gene regulation during cell reprogramming. Nat Genet. 2018;50(2):238-49. Available from: http://www.nature.com/articles/s41588-017-0030-7. https://doi.org/10.1038/s41588-017-0030-7.

21. Tapial J, Ha KCH, Sterne-Weiler T, Gohr A, Braunschweig U, Hermoso-Pulido A, et al. An atlas of alternative splicing profiles and functional associations reveals new regulatory programs and genes that simultaneously express multiple major isoforms. Genome Res. 2017;27(10):1759-68 Available from: http://genome.cshlp.org/lookup/doi/10.1101/gr.22 0962.117.

22. Kumar L, Futschik ME. Mfuzz: A software package for soft clustering of microarray data. Bioinformation. 2007;2(1):5-7 Available from: http://www.bioinformation.net/002/000200022007.htm.

23. Gopalakrishnan S, Van Emburgh BO, Shan J, Su Z, Fields CR, Vieweg J, et al. A novel DNMT3B splice variant expressed in tumor and pluripotent cells modulates genomic DNA methylation patterns and displays altered DNA binding. Mol Cancer Res. 2009;7(10):1622-34 Available from: http://mcr.aacrjournals.org/cgi/doi/10.1158/1541-7786.MCR-09-0018

24. Gopalakrishna-Pillai S, Iverson LE. A DNMT3B alternatively spliced exon and encoded peptide are novel biomarkers of human pluripotent stem cells. Pera M, editor. PLoS One. 2011;6(6):e20663 Available from: https://dx.plos.org/10.1371/ journal.pone.0020663.

25. Robinson MD, McCarthy DJ, Smyth GK. edgeR: A Bioconductor package for differential expression analysis of digital gene expression data. Bioinformatics. 2009;26(1):139-40 Available from: https://academic.oup.com/bioinformatics/articlelookup/doi/10.1093/bioinformatics/btp616.

26. Pan Q, Shai O, Misquitta C, Zhang W, Saltzman AL, Mohammad N, et al. Revealing global regulatory features of mammalian alternative splicing using a quantitative microarray platform. Mol Cell. 2004;16(6):929-41. Available from: https://linkinghub.elsevier.com/retrieve/pii/S1097276504007610. https://doi.org/10.1016/j.molcel.2004.12.004.

27. Danckwardt S, Hentze MW, Kulozik AE. 3' end mRNA processing: Molecular mechanisms and implications for health and disease. EMBO J. 2008;27(3):482-98 Available from: http://emboj.embopress.org/cgi/doi/10.1038/sj.emboj.7601932.

28. Misra A, Ou J, Zhu LJ, Green MR. Global analysis of CPSF2-mediated alternative splicing: Integration of global iCLIP and transcriptome profiling data. Genomics Data. 2015;6:217-21 Available from: https://linkinghub.elsevier.com/retrieve/pii/ S2213596015300507.

29. Chen SL, Zhu ZX, Yang X, Liu LL, He YF, Yang MM, et al. Cleavage and polyadenylation specific factor 1 promotes tumor progression via alternative polyadenylation and splicing in hepatocellular carcinoma. Front Cell Dev Biol. 2021;9(March):1-13.

30. Stadtfeld M, Maherali N, Borkent M, Hochedlinger K. A reprogrammable mouse strain from gene-targeted embryonic stem cells. Nat Methods. 2010;7(1):53-5. Available from: http://www.nature.com/articles/nmeth.1409. https://doi.org/10.1 038/nmeth.1409. 
31. Kyburz A, Friedlein A, Langen $H$, Keller W. Direct Interactions between Subunits of CPSF and the U2 snRNP contribute to the coupling of pre-mRNA 3' end processing and splicing. Mol Cell. 2006;23(2):195-205. Available from: https:// linkinghub.elsevier.com/retrieve/pii/S1097276506003790. https://doi.org/10.1016/j.molcel.2006.05.037.

32. Misra A, Ou J, Zhu LJ, Green MR. Global promotion of alternative internal exon usage by mrna 3' end formation factors. Mol Cell. 2014;58(5):819-31 Available from: https://linkinghub.elsevier.com/retrieve/pii/S1097276515001811.

33. Larson A, Fair BJ, Pleiss JA. Interconnections between RNA-processing pathways revealed by a sequencing-based genetic screen for pre-mRNA splicing mutants in fission yeast. G3. 2016;6(6):1513-23 Available from: https://academic. oup.com/g3journal/article/6/6/1513/6029966.

34. Polo JM, Anderssen E, Walsh RM, Schwarz BA, Nefzger CM, Lim SM, et al. A molecular roadmap of reprogramming somatic cells into iPS cells. Cell. 2012;151(7):1617-32. Available from: https://inkinghub.elsevier.com/retrieve/pii/S0092 867412014249. https://doi.org/10.1016/j.cell.2012.11.039.

35. Förch P, Puig O, Kedersha N, Martínez C, Granneman S, Séraphin B, et al. The apoptosis-promoting factor TIA-1 is a regulator of alternative pre-mRNA splicing. Mol Cell. 2000;6(5):1089-98. Available from: https://inkinghub.elsevier.com/ retrieve/pii/S1097276500001076. https://doi.org/10.1016/S1097-2765(00)00107-6.

36. Förch P, Puig O, Martínez C, Séraphin B, Valcárcel J. The splicing regulator TIA-1 interacts with U1-C to promote U1 snRNP recruitment to 5' splice sites. EMBO J. 2002;21(24):6882-92 Available from: http://emboj.embopress.org/cgi/doi/1 0.1093/emboj/cdf668.

37. Izquierdo JM, Majós N, Bonnal S, Martínez C, Castelo R, Guigó R, et al. Regulation of fas alternative splicing by antagonistic effects of TIA-1 and PTB on exon definition. Mol Cell. 2005;19(4):475-84. Available from: https://linkinghub. elsevier.com/retrieve/pii/S1097276505014188. https://doi.org/10.1016/j.molcel.2005.06.015.

38. Sánchez-Jiménez C, Izquierdo JM. T-cell intracellular antigen (TIA)-proteins deficiency in murine embryonic fibroblasts alters cell cycle progression and induces autophagy. Jade Lim H, editor. PLoS One. 2013;8(9):e75127 Available from: https://dx.plos.org/10.1371/journal.pone.0075127.

39. Gohr A, Irimia M. Matt: Unix tools for alternative splicing analysis. Valencia A, editor. Bioinformatics. 2019;35(1):130-2 Available from: https://academic.oup.com/bioinformatics/article/35/1/130/5053311.

40. Amit M, Donyo M, Hollander D, Goren A, Kim E, Gelfman S, et al. Differential GC content between exons and introns establishes distinct strategies of splice-site recognition. Cell Rep. 2012;1(5):543-56 [cited 2018 Jul 20]. Available from: https://linkinghub.elsevier.com/retrieve/pii/S2211124712000988.

41. Eden E, Navon R, Steinfeld I, Lipson D, Yakhini Z. GOrilla: a tool for discovery and visualization of enriched GO terms in ranked gene lists. BMC Bioinformatics. 2009;10(1):48 Available from: https://bmcbioinformatics.biomedcentral.com/a rticles/10.1186/1471-2105-10-48.

42. Ha KCH, Blencowe BJ, Morris Q. QAPA: A new method for the systematic analysis of alternative polyadenylation from RNA-seq data. Genome Biol. 2018;19(1):45 Available from: https:/genomebiology.biomedcentral.com/articles/10.1186/ s13059-018-1414-4.

43. Sardina JL, Collombet S, Tian TV, Gómez A, Di Stefano B, Berenguer C, et al. Transcription factors drive Tet2-mediated enhancer demethylation to reprogram cell fate. Cell Stem Cell. 2018;23(5):727-741.e9 Available from: https://linkinghub. elsevier.com/retrieve/pii/S1934590918304016.

44. Lewis BP, Green RE, Brenner SE. Evidence for the widespread coupling of alternative splicing and nonsense-mediated mRNA decay in humans. Proc Natl Acad Sci. 2003;100(1):189-92 Available from: http://www.pnas.org/cgi/doi/10.1073/ pnas. 0136770100.

45. Polo SE, Blackford AN, Chapman JR, Baskcomb L, Gravel S, Rusch A, et al. Regulation of DNA-end resection by hnRNPU-like proteins promotes DNA double-strand break signaling and repair. Mol Cell. 2012;45(4):505-16. Available from: https://linkinghub.elsevier.com/retrieve/pii/S1097276512000457. https://doi.org/10.1016/j.molcel.2 011.12 .035 .

46. Choi HS, Kim W-T, Kim H, Kim J-J, Ko J-Y, Lee S-W, et al. Identification and characterization of adenovirus early region 1B-associated protein 5 as a surface marker on undifferentiated human embryonic stem cells. Stem Cells Dev. 2011; 20(4):609-20 Available from: https://www.liebertpub.com/doi/10.1089/scd.2010.0265.

47. Blackwell DL, Fraser SD, Caluseriu O, Vivori C, Gordon PMK, Tyndall AV, et al. Hnrnpul1 loss of function affects skeletal and limb development. bioRxiv. 2020.

48. Kedersha NL, Gupta M, Li W, Miller I, Anderson P. RNA-binding proteins Tia-1 and Tiar link the phosphorylation of Eif-2a to the assembly of mammalian stress granules. J Cell Biol. 1999;147(7):1431-42. Available from: https:// rupress.org/jcb/article/147/7/1431/20409/RNABinding-Proteins-Tia1-and-Tiar-Link-the. https://doi.org/10.1083/jcb.147. 7.1431.

49. Piecyk M, Wax S, Beck ARP, Kedersha N, Gupta M, Maritim B, et al. TIA-1 is a translational silencer that selectively regulates the expression of TNF-a. EMBO J. 2000;19(15):4154-63 Available from: http://emboj.embopress.org/cgi/doi/10.1 093/emboj/19.15.4154.

50. Wang I, Hennig J, Jagtap PKA, Sonntag M, Valcárcel J, Sattler M. Structure, dynamics and RNA binding of the multidomain splicing factor TIA-1. Nucleic Acids Res. 2014;42(9):5949-66. Available from: http://academic.oup.com/nar/a rticle/42/9/5949/1253634/Structure-dynamics-and-RNA-binding-of-the. https://doi.org/10.1093/nar/gku193.

51. Wang Z, Kayikci M, Briese M, Zarnack K, Luscombe NM, Rot G, et al. iCLIP Predicts the dual splicing effects of TIA-RNA interactions. Misteli T, editor. PLoS Biol. 2010;8(10):e1000530 Available from: https://dx.plos.org/10.1371/journal.pbio.1 000530.

52. Ritschka B, Storer M, Mas A, Heinzmann F, Ortells MC, Morton JP, et al. The senescence-associated secretory phenotype induces cellular plasticity and tissue regeneration. Genes Dev. 2017;31(2):172-83 Available from: http://genesdev.cshlp. org/lookup/doi/10.1101/gad.290635.116.

53. Mosteiro L, Pantoja C, Alcazar N, Marión RM, Chondronasiou D, Rovira M, et al. Tissue damage and senescence provide critical signals for cellular reprogramming in vivo. Science (80- ). 2016;354(6315):aaf4445 Available from: https://www. sciencemag.org/lookup/doi/10.1126/science.aaf4445.

54. Brady JJ, Li M, Suthram S, Jiang H, Wong WH, Blau HM. Early role for IL-6 signalling during generation of induced pluripotent stem cells revealed by heterokaryon RNA-Seq. Nat Cell Biol. 2013;15(10):1244-52. Available from: http:// www.nature.com/articles/ncb2835. https://doi.org/10.1038/ncb2835. 
55. Stelzer G, Rosen N, Plaschkes I, Zimmerman S, Twik M, Fishilevich S, et al. The GeneCards Suite: from gene data mining to disease genome sequence analyses. Curr Protoc Bioinforma. 2016;54(1):1.30.1-1.30.33 Available from: https:// onlinelibrary.wiley.com/doi/10.1002/cpbi.5.

56. Travis A, Amsterdam A, Belanger C, Grosschedl R. LEF-1, a gene encoding a lymphoid-specific protein with an HMG domain, regulates T-cell receptor alpha enhancer function [corrected]. Genes Dev. 1991;5(5):880-94 Available from: http://www.genesdev.org/cgi/doi/10.1101/gad.5.5.880.

57. Waterman ML, Fischer WH, Jones KA. A thymus-specific member of the HMG protein family regulates the human T cell receptor Ca enhancer. Genes Dev. 1991;5(4):656-69 Available from: http://www.genesdev.org/cgi/doi/10.1101/gad.5.4.656.

58. Oosterwegel M, van de Wetering M, Timmerman J, Kruisbeek A, Destree O, Meijlink F, et al. Differential expression of the HMG box factors TCF-1 and LEF-1 during murine embryogenesis. Development. 1993;1 18(2):439-48 Available from: http://www.ncbi.nlm.nih.gov/pubmed/8223271.

59. van Genderen C, Okamura RM, Farinas I, Quo RG, Parslow TG, Bruhn L, et al. Development of several organs that require inductive epithelial-mesenchymal interactions is impaired in LEF-1-deficient mice. Genes Dev. 1994;8(22):2691-703 Available from: http://www.genesdev.org/cgi/doi/10.1101/gad.8.22.2691.

60. Zhou P, Byrne C, Jacobs J, Fuchs E. Lymphoid enhancer factor 1 directs hair follicle patterning and epithelial cell fate. Genes Dev. 1995;9(6):700-13 Available from: http://www.genesdev.org/cgi/doi/10.1101/gad.9.6.700.

61. Mallory MJ, Jackson J, Weber B, Chi A, Heyd F, Lynch KW. Signal- and development-dependent alternative splicing of LEF1 in T cells is controlled by CELF2. Mol Cell Biol. 2011;31(11):2184-95 Available from: http://mcb.asm.org/cgi/doi/10.1128/MCB.05170-11.

62. Mallory MJ, Allon SJ, Qiu J, Gazzara MR, Tapescu I, Martinez NM, et al. Induced transcription and stability of CELF2 mRNA drives widespread alternative splicing during T-cell signaling. Proc Natl Acad Sci U S A. 2015;112(17):E2139-48 Available from: http://www.pnas.org/lookup/doi/10.1073/pnas.1423695112.

63. Ajith S, Gazzara MR, Cole BS, Shankarling G, Martinez NM, Mallory MJ, et al. Position-dependent activity of CELF2 in the regulation of splicing and implications for signal-responsive regulation in T cells. RNA Biol. 2016;13(6):569-81 Available from: https:/www.tandfonline.com/doi/full/10.1080/15476286.2016.1176663.

64. Bruhn L, Munnerlyn A, Grosschedl R. ALY, a context-dependent coactivator of LEF-1 and AML-1, is required for TCRa enhancer function. Genes Dev. 1997;11(5):640-53 Available from: http://www.genesdev.org/cgi/doi/10.1101/gad.11.5.640.

65. Levanon D, Goldstein RE, Bernstein Y, Tang H, Goldenberg D, Stifan S, et al. Transcriptional repression by AML1 and LEF1 is mediated by the TLE/Groucho corepressors. Proc Natl Acad Sci U S A. 1998;95(20):11590-5 Available from: http:// www.pnas.org/cgi/doi/10.1073/pnas.95.20.11590.

66. Jesse S, Koenig A, Ellenrieder V, Menke A. Lef-1 isoforms regulate different target genes and reduce cellular adhesion. Int J Cancer. 2010;126(5):1109-20 Available from: http://doi.wiley.com/10.1002/ijc.24802.

67. Rodriguez-Ubreva J, Ciudad L, van Oevelen C, Parra M, Graf T, Ballestar E. C/EBPa-mediated activation of MicroRNAs 34a and 223 inhibits Lef1 expression to achieve efficient reprogramming into macrophages. Mol Cell Biol. 2014;34(6):114557 Available from: http://mcb.asm.org/cgi/doi/10.1128/MCB.01487-13.

68. Edgar R. Gene Expression Omnibus: NCBI gene expression and hybridization array data repository. Nucleic Acids Res. 2002;30(1):207-10 Available from: https://academic.oup.com/nar/article-lookup/doi/10.1093/nar/30.1.207.

69. Dobin A, Davis CA, Schlesinger F, Drenkow J, Zaleski C, Jha S, et al. STAR: ultrafast universal RNA-seq aligner. Bioinformatics. 2013;29(1):15-21 Available from: https:/academic.oup.com/bioinformatics/article-lookup/doi/10.1093/bioinformatics/bts635.

70. McCarthy DJ, Chen Y, Smyth GK. Differential expression analysis of multifactor RNA-Seq experiments with respect to biological variation. Nucleic Acids Res. 2012;40(10):4288-97. Available from: https://academic.oup.com/nar/article/40/1 0/4288/2411520. https://doi.org/10.1093/nar/gks042.

71. Irimia M, Weatheritt RJ, Ellis JD, Parikshak NN, Gonatopoulos-Pournatzis T, Babor M, et al. A highly conserved program of neuronal microexons is misregulated in autistic brains. Cell. 2014;159(7):1511-23. Available from: https://linkinghub. elsevier.com/retrieve/pii/S0092867414015128. https://doi.org/10.1016/j.cell.2014.11.035.

72. Futschik ME, Carlisle B. Noise-robust soft clustering of gene expression time-course data. J Bioinform Comput Biol. 2005; 3(4):965-88 Available from: https://www.worldscientific.com/doi/abs/10.1142/S0219720005001375.

73. Yeo G, Burge CB. Maximum entropy modeling of short sequence motifs with applications to RNA splicing signals. J Comput Biol. 2004;11(2-3):377-94 Available from: http://www.liebertpub.com/doi/10.1089/1066527041410418.

74. Ray D, Kazan H, Cook KB, Weirauch MT, Najafabadi HS, Li X, et al. A compendium of RNA-binding motifs for decoding gene regulation. Nature. 2013;499(7457):172-7. Available from: http://www.nature.com/nature/journal/v499/n7457/abs/ nature12311.html\#supplementary-information. https://doi.org/10.1038/nature12311.

75. Chen H, Boutros PC. VennDiagram: a package for the generation of highly-customizable Venn and Euler diagrams in R. BMC bioinformatics. 2011;12(1):1-7.

76. Supek F, Bošnjak M, Škunca N, Šmuc T. REVIGO summarizes and visualizes long lists of gene ontology terms. Gibas C, editor. PLoS One. 2011;6(7):e21800 Available from: https://dx.plos.org/10.1371/journal.pone.0021800.

77. Patro R, Duggal G, Love Ml, Irizarry RA, Kingsford C. Salmon provides fast and bias-aware quantification of transcript expression. Nat Methods. 2017;14(4):417-9. Available from: http://www.nature.com/articles/nmeth.4197. https://doi.org/1 0.1038/nmeth.4197.

78. Quinlan AR, Hall IM. BEDTools: a flexible suite of utilities for comparing genomic features. Bioinformatics. 2010;26(6):8412 Available from: https://academic.oup.com/bioinformatics/article-lookup/doi/10.1093/bioinformatics/btq033.

79. Di Stefano B, Graf T. Very rapid and efficient generation of induced pluripotent stem cells from mouse pre-B Cells. In: Methods in Molecular Biology; 2016. p. 45-56. Available from: http://link.springer.com/10.1007/7651_2014_133.

80. Gibson DG, Young L, Chuang RY, Venter JC, Hutchison CA, Smith HO. Enzymatic assembly of DNA molecules up to several hundred kilobases. Nat Methods. 2009;6(5):343-5. Available from: https://www.nature.com/articles/nmeth.131 8\#supplementary-information. https://doi.org/10.1038/nmeth.1318.

81. Valcárcel J, Vivori C, Papasaikas P, Stadhouders R, Di Stefano B, Ribó Rubio A, Berenguer Balaguer C, Generoso S, Mallol A, Sardina JL, Payer B, Graf T. Dynamics of alternative splicing during somatic cell reprogramming reveals functions for RNA-binding proteins CPSF3, hnRNP UL1 and TIA1. Datasets. Gene Expression Omnibus. 2021. https://www.ncbi.nlm.nih. gov/geo/query/acc.cgi?acc=GSE158633.

82. Valcárcel J, Vivori C, Papasaikas P, Stadhouders R, Di Stefano B, Ribó Rubio A, Berenguer Balaquer C, Generoso S, Mallol A, Sardina JL, Payer B, Graf T. Dynamics of alternative splicing during somatic cell reprogramming reveals functions for 
RNA-binding proteins CPSF3, hnRNP UL1 and TIA1. Github. Repository. 2021. https://doi.org/10.5281/zenodo.4724548 and https://github.com/cvivori/AS-reprogramming-KD-OE.

83. Valcárcel J, Vivori C, Papasaikas P, Stadhouders R, Di Stefano B, Ribó Rubio A, Berenguer Balaguer C, Generoso S, Mallol A, Sardina JL, Payer B, Graf T. Dynamics of alternative splicing during somatic cell reprogramming reveals functions for RNA-binding proteins CPSF3, hnRNP UL1 and TIA1. Github. Repository. 2021. https://doi.org/10.5281/zenodo.4723460 and https://github.com/cvivori/AS-BandMEF-reprogramming.

\section{Publisher's Note}

Springer Nature remains neutral with regard to jurisdictional claims in published maps and institutional affiliations.

Ready to submit your research? Choose BMC and benefit from:

- fast, convenient online submission

- thorough peer review by experienced researchers in your field

- rapid publication on acceptance

- support for research data, including large and complex data types

- gold Open Access which fosters wider collaboration and increased citations

- maximum visibility for your research: over 100M website views per year

At $\mathrm{BMC}$, research is always in progress.

Learn more biomedcentral.com/submissions 